This item was submitted to Loughborough's Research Repository by the author.

Items in Figshare are protected by copyright, with all rights reserved, unless otherwise indicated.

\title{
Multidimensional Baker-Akhiezer functions and Huygens' principle
}

PLEASE CITE THE PUBLISHED VERSION

LICENCE

CC BY-NC-ND 4.0

\section{REPOSITORY RECORD}

Chalykh, O.A., Mikhail V. Feigin, and A.P. Veselov. 2019. "Multidimensional Baker-akhiezer Functions and Huygens' Principle”. figshare. https://hdl.handle.net/2134/828. 


\title{
Multidimensional Baker-Akhiezer Functions and Huygens' Principle
}

\author{
O.A.Chalykh $^{\dagger}$, M.V.Feigin ${ }^{\dagger, \S}$, A.P.Veselov ${ }^{\ddagger, \star}$ \\ $\dagger$ Department of Mathematics and Mechanics, Moscow State University, \\ Moscow, 119899, Russia \\ $\S$ Independent University of Moscow, Bolshoy Vlasevsky per. 11, Moscow, 121002, Russia \\ $\ddagger$ Department of Mathematical Sciences, Loughborough University, \\ Loughborough, LE11 3TU, UK \\ * Landau Institute for Theoretical Physics, Kosygina 2, Moscow, 117940, Russia \\ E-mail addresses: chalykh@nw.math.msu.su,mfeigin@dnttm.ru, A.P.Veselov@lboro.ac.uk
}

\begin{abstract}
A notion of rational Baker-Akhiezer (BA) function related to a configuration of hyperplanes in $\mathbf{C}^{\mathbf{n}}$ is introduced. It is proved that BA function exists only for very special configurations (locus configurations), which satisfy certain overdetermined algebraic system. The BA functions satisfy some algebraically integrable Schrödinger equations, so any locus configuration determines such an equation. Some results towards the classification of all locus configurations are presented. This theory is applied to the famous Hadamard's problem of description of all hyperbolic equations satisfying Huygens' Principle. We show that in a certain class all such equations are related to locus configurations and the corresponding fundamental solutions can be constructed explicitly from the BA functions.
\end{abstract}

\section{Introduction}

The notion of Baker-Akhiezer function (BA function) has been introduced by Krichever [1] in the theory of finite-gap or algebro-geometric solutions of the nonlinear PDE's, integrable by the inverse scattering method [2]. The BA function is a far-going generalisation of the classical function

$$
\psi=\frac{\sigma(x-z)}{\sigma(x) \sigma(z)} e^{\zeta(z) x}
$$

well-known as a solution to the classical Lame equation:

$$
L \psi=\lambda \psi, \quad L=-\frac{d^{2}}{d x^{2}}+2 \wp(x), \quad \lambda=-\wp(z) .
$$

Here $\sigma, \zeta$ and $\wp$ are classical Weierstrass elliptic functions (see e.g. [3]). 
In the degenerate case one has the corresponding trigonometric and rational versions:

$$
\begin{gathered}
\psi_{\text {trig }}=\left(1-\frac{1}{k} \cot x\right) e^{k x}, \quad L=-\frac{d^{2}}{d x^{2}}+\frac{2}{\sin ^{2} x}, \\
\psi_{\text {rat }}=\left(1-\frac{1}{k x}\right) e^{k x}, \quad L=-\frac{d^{2}}{d x^{2}}+\frac{2}{x^{2}} .
\end{gathered}
$$

Certain multidimensional versions of these functions in the rational and trigonometric cases have been introduced by Chalykh and Veselov in [4] in the theory of quantum Calogero-Moser problem. In this paper we will restrict ourselves by the rational case only. The construction of [4] (see also [5]) relates such a BA function $\psi$ to a configuration $\mathfrak{A}$ of the hyperplanes $\Pi_{\alpha}$ in a complex Euclidean space $\mathbf{C}^{n}$ given by the equations $(\alpha, x)=0$, taken with some multiplicities $m_{\alpha} \in \mathbf{Z}_{+}$. Here $\alpha \in \mathcal{A}, \mathcal{A}$ is a finite set of noncollinear vectors. The function $\psi(k, x), k, x \in \mathbf{C}^{n}$ is determined by certain analytic properties in $k$ (see Section 1) and exists only for very special configurations.

The most important property of the BA function is that it is an eigenfunction of the multidimensional algebraically integrable Schrödinger operator $L$, which in our case has the form

$$
L=-\Delta+\sum_{\alpha \in \mathcal{A}} \frac{m_{\alpha}\left(m_{\alpha}+1\right)(\alpha, \alpha)}{(\alpha, x)^{2}}
$$

(see $[4,5])$.

When $\mathfrak{A}$ is a Coxeter configuration, i.e. $\mathfrak{A}$ consists of the reflection hyperplanes for some finite reflection group $W$ with $W$-invariant multiplicities, then the corresponding operator $L$ is the Hamiltonian of the generalised quantum Calogero-Moser problem (after Olshanetsky and Perelomov [6]) with special integer-valued parameters. The existence of the BA function in this case was proved in [5] with the help of Heckman's result [7].

At that time it was believed that the Coxeter case is the only one, when $\psi$ does exist, but it turned out to be not the case. The first non-Coxeter examples have been found by the authors in [8] (see also [9]).

According to the general procedure proposed by Berest and Veselov in [10] this led to the new examples of the hyperbolic equations satisfying the Huygens' Principle in Hadamard's sense. Motivated by these results Berest and Lutsenko started the investigation of the case when the potential depends on two coordinates only and found other new examples of the huygensian equations [11]. Later Berest proved [12] that they have actually found all such equations under assumption that the potential is homogeneous of degree $(-2)$. Since a generic Berest-Lutsenko potential could not be described by the construction [4], this was the reason for us to revise it.

In the Section 1 we give such a revised definition of the BA function, which can be derived from the corresponding Schrödinger equation and therefore covers all the possible cases. It is remarkable that there exists an effective way to check for a given configuration whether BA function exists or not. Namely, as we prove in Sections 2 and 3, the following overdetermined system of algebraic equations is a necessary and sufficient condition for the existence of the Baker-Akhiezer function:

$$
\sum_{\substack{\beta \in \mathcal{A} \\ \beta \neq \alpha}} \frac{m_{\beta}\left(m_{\beta}+1\right)(\beta, \beta)(\alpha, \beta)^{2 j-1}}{(\beta, x)^{2 j+1}} \equiv 0 \text { on the hyperplane }(\alpha, x)=0
$$


for each $\alpha \in \mathcal{A}$ and $j=1,2, \ldots, m_{\alpha}$.

They are equivalent to the vanishing of the first $m_{\alpha}$ odd terms in the Laurent expansion of the corresponding potential

$$
u(x)=\sum_{\alpha \in \mathcal{A}} \frac{m_{\alpha}\left(m_{\alpha}+1\right)(\alpha, \alpha)}{(\alpha, x)^{2}}
$$

at the hyperplane $(\alpha, x)=0$. Similar characterisation of the rational finite-gap potentials in one dimension has been first proposed in the famous paper [13] by Airault, McKean and Moser, who introduced the term "locus" in this situation. We will also use this terminology, calling the equations (2) as well as its general affine version (see below) as locus equations. Duistermaat and Grünbaum [14] discovered the interpretation of such equations as a trivial monodromy condition for the corresponding one-dimensional Schrödinger equation in the complex domain. We give a similar interpretation for our locus equations (2) in Section 2.

However, to describe all the configurations, satisfying the locus equations (2) (the locus configurations) seems to be a very difficult problem. At the moment it is solved only in dimension 2, where the answer is given by the Berest-Lutsenko construction. In dimension $n>2$ all known examples of the locus configurations are the Coxeter configurations and their special "deformations" [8],[9]. In the section 4 we present all the results, which are known in this direction so far.

The generalisation of our construction to the affine configurations of the hyperplanes is discussed in the section 5 . The potential $u$ and the locus equations in that case have the form:

$$
\begin{gathered}
u(x)=\sum_{i=1}^{K} \frac{m_{i}\left(m_{i}+1\right)\left(\alpha_{i}, \alpha_{i}\right)}{\left(\left(\alpha_{i}, x\right)+c_{i}\right)^{2}} \\
\sum_{j \neq i} \frac{m_{j}\left(m_{j}+1\right)\left(\alpha_{j}, \alpha_{j}\right)\left(\alpha_{i}, \alpha_{j}\right)^{2 s-1}}{\left(\left(\alpha_{j}, x\right)+c_{j}\right)^{2 s+1}} \equiv 0
\end{gathered}
$$

identically on the hyperplane $\left(\alpha_{i}, x\right)+c_{i}=0$ for all $i=1, \ldots, K$ and $s=1, \ldots, m_{i}$. Unfortunately, so far a little is known about the affine locus configurations, which are not linear, i.e. with not all the hyperplanes to pass through one point. Apart from the one-dimensional case investigated in [13], [15], there are only some reducible examples discovered by Berest and Winternitz [16]. In fact, we show that the classification problem for the affine locus configurations can be reduced to the linear case (2) by the isotropic projectivisation procedure.

In the last section we discuss the relations of our BA function $\psi$ and locus configurations to the Huygens' Principle. The main result says that for any locus configuration in dimension $n$ the corresponding hyperbolic equation

$$
\left(\square_{N+1}+u\left(x_{1}, \ldots, x_{n}\right)\right) \phi=0
$$

satisfies Huygens' Principle for large enough odd $N$. Conversely, we show that if the equation (6) satisfies Huygens' Principle and all the Hadamard's coefficients are rational functions, then $u(x)$ has a form (4) for some locus configuration. 
We conjecture that this construction gives all huygensian equations of the form $\left(\square_{N+1}+\right.$ $\left.u\left(x_{1}, \ldots, x_{n}\right)\right) \phi=0$. In the case $n=1$ it is well-known result by Stellmacher and Lagnese [17]. When $n=2$ and $u$ is homogeneous this follows from Berest's theorem [12]. The proof of the general case would lead to the solution of the famous Hadamard's problem in the class (6).

\section{Rational Baker-Akhiezer function related to a con- figuration of hyperplanes.}

Let $\mathcal{A}$ be a finite set of noncollinear vectors $\alpha=\left(\alpha_{1}, \ldots, \alpha_{n}\right) \in \mathbf{C}^{n}$ with multiplicities $m_{\alpha} \in \mathbf{N}$. We will assume that $(\alpha, \alpha)=\sum_{i=1}^{n} \alpha_{i}^{2} \neq 0$.

Definition. A function $\psi(k, x), k, x \in \mathbf{C}^{n}$ will be called Baker-Akhiezer function (BA function), if the following two conditions are fulfilled:

1) $\psi(k, x)$ has a form

$$
\psi(k, x)=\frac{P(k, x)}{A(k)} e^{(k, x)},
$$

where $A(k)=\prod_{\alpha \in \mathcal{A}}(k, \alpha)^{m_{\alpha}}, P(k, x)$ is a polynomial in $k$ with the highest term $A(k)$;

2) for all $\alpha \in \mathcal{A}$

$$
\partial_{\alpha}\left(\psi(k, x)(k, \alpha)^{m_{\alpha}}\right)=\partial_{\alpha}^{3}\left(\psi(k, x)(k, \alpha)^{m_{\alpha}}\right)=\ldots=\partial_{\alpha}^{2 m_{\alpha}-1}\left(\psi(k, x)(k, \alpha)^{m_{\alpha}}\right) \equiv 0
$$

on the hyperplane $\Pi_{\alpha}:(k, \alpha)=0$, where $\partial_{\alpha}=\left(\alpha, \frac{\partial}{\partial k}\right)$ is the normal derivative for this hyperplane.

Notice that (7) means that $\psi$ is a rational function of $k$ with the prescribed poles along the hyperplanes $\Pi_{\alpha}, \alpha \in \mathcal{A}$ and with the asymptotic behaviour at infinity:

$$
\psi=(1+o(1)) e^{(k, x)}
$$

when $k \rightarrow \infty$ along the rays outside the singularities (cf.[1]).

First of all, in the same way as in [4],[5] one can prove the following

Theorem 1.1. If the Baker-Akhiezer function $\psi$ exists then it is unique and satisfies the algebraically integrable Schrödinger equation

$$
L \psi=-k^{2} \psi
$$

where

$$
L=-\Delta+\sum_{\alpha \in \mathcal{A}} \frac{m_{\alpha}\left(m_{\alpha}+1\right)(\alpha, \alpha)}{(\alpha, x)^{2}}
$$

Algebraic integrability of the operator (10) means that $L$ is a part of a rich (supercomplete) commutative ring of partial differential operators (see [5] for precise definitions). This ring is described by the following theorem.

Theorem 1.2. Let $\mathcal{R}_{\mathcal{A}}$ be the ring of polynomials $f(k)$ satisfying the following properties

$$
\partial_{\alpha} f(k)=\partial_{\alpha}^{3} f(k)=\ldots=\partial_{\alpha}^{2 m_{\alpha}-1} f(k) \equiv 0
$$


on the hyperplane $(\alpha, k)=0$ for any $\alpha \in \mathcal{A}$.

If the Baker-Akhiezer function $\psi(k, x)$ exists then for any polynomial $f(k) \in \mathcal{R}_{\mathcal{A}}$ there exists some differential operator $L_{f}\left(x, \frac{\partial}{\partial x}\right)$ such that

$$
L_{f} \psi(k, x)=f(k) \psi(k, x)
$$

All such operators form a commutative ring isomorphic to the ring $\mathcal{R}_{\mathcal{A}}$. The Schrödinger operator (10) corresponds to $f(k)=-k^{2}$.

We give the proof of these statements in a more general affine situation in the Section 5 .

We should note that there exists the following explicit formula for $L_{f}$ (due to Yu.Berest [18]).

Theorem 1.3. The commuting partial differential operators $L_{f}$ for $f \in \mathcal{R}_{\mathcal{A}}$ are given by the formula

$$
L_{f}=c_{N}\left(a d_{L}\right)^{N}[\hat{f}(x)]
$$

where $c_{N}=(-1)^{N} / 2^{N} N$ !, $N=\operatorname{deg} f, \hat{f}$ is the operator of multiplication by $f(x)$, and $\left(a d_{L}\right)^{N}$ means the $N$-th iteration of the standard ad-procedure, $a d_{A} B=A B-B A$.

The proof follows from the results of the next section (see Corollary 2.5).

We should note that originally in [4] another axiomatics for the $\psi$ - function was proposed. There was considered a function $\phi(k, x)$ of the form

$$
\phi(k, x)=P(k, x) e^{(k, x)},
$$

where $P(k, x)$, as in $(7)$, is a polynomial in $k$ with the highest term $A(k)$, with the property

$$
\partial_{\alpha}(\phi(k, x))=\partial_{\alpha}^{3}(\phi(k, x))=\ldots=\partial_{\alpha}^{2 m_{\alpha}-1}(\phi(k, x)) \equiv 0
$$

at the hyperplane $\Pi_{\alpha}$.

Comparing (13), (14) with (7), (8) we see that the difference between these two axiomatics is due to the additional factor $\prod_{\beta \neq \alpha}(k, \beta)^{m_{\beta}}$. In the Coxeter situation considered in [4] (see Section 4 below) this factor is not essential because of its symmetry.

It turns out that this minor change makes the axiomatics less restrictive and leads to a richer class of the integrable Schrödinger operators. We will prove (see Corollary 2.7) that if there exists $\phi$ satisfying the conditions (13), (14) then there exists also the BA function $\psi$ with the properties (7), (8) and in that case $\psi=\frac{\phi}{A(k)}$. Converse is not true: there are configurations, for which $\psi$ does exist but $\phi$ does not (see remark after the proof of the Theorem 4.4).

\section{Monodromy and BA functions.}

Let $L=-\Delta+u(x)$ be a Schrödinger operator with a meromorphic potential $u(x)$ having a pole along the hyperplane $\Pi_{\alpha}:(\alpha, x)=0$, which is assumed to be non-isotropic: $(\alpha, \alpha) \neq 0$. 
We are looking for a formal solution $\phi$ of the Schrödinger equation $L \phi=\lambda \phi$ in the form

$$
\phi(x)=\sum_{s \geq 0} \phi_{s}^{(\alpha)}(\alpha, x)^{\mu+s}
$$

for some $\mu$, where the coefficients $\phi_{s}^{(\alpha)}=\phi_{s}^{(\alpha)}\left(x^{\perp}\right)$ are some analytic functions on the hyperplane $\Pi_{\alpha}, x^{\perp}$ is orthogonal projection of $x$ onto $\Pi_{\alpha}, \phi_{0}^{(\alpha)} \neq 0$.

Let's suppose that the equation $L \phi=\lambda \phi$ has a solution of the form (15) with some $\mu<0$. Then the substitution into the equation gives immediately that the potential $u(x)$ must have a second order pole along $\Pi_{\alpha}$ : the Laurent expansion in the normal direction $\alpha$ has the form

$$
u(x)=\sum_{k \geq-2} c_{k}^{(\alpha)}(\alpha, x)^{k}
$$

with $c_{-2}^{(\alpha)}=\mu(\mu-1)(\alpha, \alpha)$.

Moreover, we obtain the following recurrent relations for the coefficients $\phi_{s}^{(\alpha)}$ :

$$
(\alpha, \alpha)(\mu(\mu-1)-(\mu+s)(\mu+s-1)) \phi_{s}=(\widetilde{\Delta}+\lambda) \phi_{s-2}-\sum_{i=-1}^{s-2} c_{i} \phi_{s-i-2}
$$

$(s=1,2, \ldots)$, where $\widetilde{\Delta}$ is the Laplacian $\Delta$ restricted to the hyperplane $\Pi_{\alpha}$ and we omitted all the indices $\alpha$ in the coefficients.

If $2 \mu \notin \mathbf{Z}$ we can determine all $\phi_{s}$ from (17) and obtain the solution (15) starting from an arbitrary function $\phi_{0}$ (the same procedure gives also another solution with $\mu^{\prime}=$ $-1-\mu)$.

In the one-dimensional case this is a classical way (going back to Frobenius, see e.g. [27]) to construct the basis of solutions of the corresponding equation

$$
-\varphi^{\prime \prime}+u(x) \varphi=\lambda \varphi
$$

in a vicinity of its regular singular point. In the case when the equation (18) has no monodromy in the complex domain, i.e. all the solutions are single-valued, we have that

1) $\mu$ must be an integer: $\mu=-m, m \in \mathbf{Z}_{+}$,

2) the first $2 m+1$ equations from (17) must be compatible.

In case if this is true for each energy level $\lambda$ we will say that the Schrödinger operator has trivial monodromy.

In the multidimensional case there exists a generalisation of Frobenius's theory for the partial differential equations with the regular singularities in the complex domain (see [28]). For the Schrödinger equation with a singularity along a hypersurface the regularity condition means that the potential has a second order pole at most.

The considerations above motivate the following

Definition. We say that a Schrödinger operator $L=-\Delta+u(x)$ with meromorphic potential $u(x)$ having a second order pole along the hyperplane $\Pi_{\alpha}:(\alpha, x)=0$ has local trivial monodromy around this hyperplane if 
1) the Laurent coefficient $c_{-2}^{(\alpha)}$ in the expansion (16) has the form $c_{-2}^{(\alpha)}=m_{\alpha}\left(m_{\alpha}+\right.$ 1) $(\alpha, \alpha)$ for some $m_{\alpha} \in \mathbf{Z}_{+}$,

2) the system (17) with $\mu=-m_{\alpha}$ is compatible for any function $\phi_{0}$ and for all $\lambda \in \mathbf{C}$.

Theorem 2.1. L has local trivial monodromy around $\Pi_{\alpha}$ if and only if the coefficients of the normal Laurent expansion of the potential $u(x)$ near $\Pi_{\alpha}$

$$
u(x)=\sum_{s \geq-2} c_{s}^{(\alpha)}(\alpha, x)^{s}
$$

satisfy the following conditions: $c_{-2}=m_{\alpha}\left(m_{\alpha}+1\right)(\alpha, \alpha)$ for some $m_{\alpha} \in \mathbf{Z}_{+}$, and

$$
c_{-1}^{(\alpha)}=c_{1}^{(\alpha)}=c_{3}^{(\alpha)}=\ldots=c_{2 m_{\alpha}-1}^{(\alpha)} \equiv 0 \text { on } \Pi_{\alpha} .
$$

In that case the Laurent expansions of the corresponding eigenfunctions $\phi$ (15) satisfy the conditions

$$
\phi_{1}^{(\alpha)}=\phi_{3}^{(\alpha)}=\ldots=\phi_{2 m_{\alpha}-1}^{(\alpha)} \equiv 0 \text { on } \Pi_{\alpha}
$$

Proof is similar to the one-dimensional case considered by J.Duistermaat and A.Grünbaum [14]. Let's demonstrate the idea in the simplest case when $m_{\alpha}=1$. After substitution (15) into the Schrödinger equation, we deduce that $\mu=2$ and derive the following recurrent relations for $\phi_{k}^{(\alpha)}$ :

$$
\left\{\begin{array}{l}
\left(-2+c_{-2}\right) \phi_{0}=0 \\
2 \phi_{1}+c_{-1} \phi_{0}=0 \\
2 \phi_{2}+(-\widetilde{\Delta}-\lambda) \phi_{0}+c_{0} \phi_{0}+c_{-1} \phi_{1}=0 \\
0 \phi_{3}+(-\widetilde{\Delta}-\lambda) \phi_{1}+c_{1} \phi_{0}+c_{0} \phi_{1}+c_{-1} \phi_{2}=0 \\
\ldots
\end{array}\right.
$$

where $\widetilde{\Delta}$ is the Laplacian $\Delta$ restricted to the hyperplane $\Pi$ (we omitted all the subindices $\alpha$ in these formulas and assumed that $(\alpha, \alpha)=1)$. These relations allow one to find all the coefficients uniquely except $\phi_{0}$ (which is an arbitrary function) and $\phi_{3}$, provided the consistency of the first four equations. From the first equation it follows that $c_{-2}=2$. Expressing $\phi_{1}$ and $\phi_{2}$ from the second and the third equations and substituting them into the fourth one we arrive at the relation

$$
(-\widetilde{\Delta}-\lambda)\left(-\frac{1}{2} c_{-1} \phi_{0}\right)-\frac{1}{2} c_{-1}(-\widetilde{\Delta}-\lambda) \phi_{0}+\left(c_{1}-c_{0} c_{-1}+\frac{1}{4} c_{-1}^{3}\right) \phi_{0}=0,
$$

which should be valid for all $\phi_{0}$ and $\lambda$. Vanishing of the leading term in $\lambda$ gives $c_{-1} \phi_{0} \equiv 0$, i.e. $c_{-1} \equiv 0$. The relation reduces after that to $c_{1} \phi_{0}=0$, thus $c_{1} \equiv 0$. Notice that as it follows from the second equation $\phi_{1}=-\frac{1}{2} c_{-1} \phi_{2} \equiv 0$. This completes the proof in the case when $m_{\alpha}=1$. In the general case one should use induction arguments (see [14], p.196).

Remark. One can consider a more general case, when $u(x)$ has a singularity along an arbitrary hypersurface $\varphi(x)=0$. However, analysis of the corresponding relations (21) shows that the hypersurface has to be a hyperplane (cf. [19]). 
Now let's consider a Schrödinger operator (1) L, corresponding to some Baker Akhiezer function $\psi$. We claim that such an operator has local trivial monodromy around all the singular hyperplanes. To prove one can consider for a given $\lambda$ the $(n-1)-$ dimensional family of the solutions of the Schrödinger equation

$$
(L-\lambda) \varphi=0
$$

of the form $\varphi=\psi(k, x)$ with $k^{2}=-\lambda$. They have proper pole behaviour near the hyperplane $(\alpha, x)=0$. Unfortunately, $\psi_{0}^{(\alpha)}$ depends on $k$ and is not an arbitrary function on the hyperplane, so we have to present additional arguments. We'll prove a slightly more general result, which we will use also in the section 6 .

Theorem 2.2. Let the Schrödinger operator $L=-\Delta+u(x)$ have an eigenfunction $\psi(k, x)$

$$
L \psi=-k^{2} \psi
$$

of the form $\psi=P(k, x) e^{(k, x)}$, where $P$ is a finite sum of some functions which are homogeneous in $k$ and meromorphic in $x$. Then the singularities of $u(x)$ are second order poles located on a union of non-isotropic hyperplanes and $L$ has local trivial monodromy around these hyperplanes.

Proof. The fact that singularities of $u(x)$ must be located on the hyperplanes was proved by $\mathrm{Yu}$.Yu. Berest and A.P. Veselov in [20] under assumption that $P$ is a polynomial in $k$, but their proof works also in the case when $P$ is a finite sum of the homogeneous in $k$ functions. The fact that these hyperplanes must be non-isotropic follows from the zero-residue lemma of the same paper [20] (see also [19]).

Let's now prove that the conditions (19) are to be satisfied. After a proper choice of orthonormal basis we may assume that the hyperplane under consideration has the equation $x_{1}=0$, and let's consider the Laurent expansion for the function $\psi(k, x)$ :

$$
\psi(k, x)=x_{1}^{-m} \sum_{i=0}^{+\infty} \psi_{i}\left(k, x_{2}, \ldots, x_{n}\right) x_{1}^{i}
$$

Let's prove first that $m$ has to be positive. Let $P^{0}$ be the highest homogeneous term of $P$, then from the Schrödinger equation we have $\sum k_{i} \partial / \partial x_{i} P^{0}=0$. So $P^{0}(k, x+k t)$ is constant while $t$ varies, hence if $P^{0}$ vanishes on the hyperplane $x_{1}=0$, then it vanishes identically. Thus, $P^{0}$ and therefore $\psi$ can not be zero at the hyperplane, so $m$ in (22) must be positive.

Substitution (22) to Schrödinger equation immediately gives that $c_{-2}=m(m+1)$ and leads to the following recurrence relations:

$$
(m(m+1)-(j+2-m)(j+1-m)) \psi_{j+2}=\left(\widetilde{\Delta}-k^{2}\right) \psi_{j}-\sum_{i=-1}^{j} c_{i} \psi_{j-i},
$$

$(j=-1,0,1,2, \ldots), \widetilde{\Delta}=\frac{\partial^{2}}{\partial x_{2}^{2}}+\ldots+\frac{\partial^{2}}{\partial x_{n}^{2}}$. To prove (19) let's suppose that $c_{-1}=c_{1}=$ $\ldots=c_{2 p-3}=0$, but $c_{2 p-1} \neq 0$ for some $p<m+1$. Considering $j=-1,1,3, \ldots, 2 p-3$ it is easy to see that $\psi_{1}=\psi_{3}=\ldots=\psi_{2 p-1}=0$. From the form of the function $\psi$ it follows that $\psi_{j}=P_{j}\left(k, x_{2}, \ldots, x_{n}\right) e^{(\tilde{k}, \tilde{x})}$, where $P_{j}$ are finite sum of homogeneous functions in $k, \tilde{k}=\left(k_{2}, \ldots, k_{n}\right), \tilde{x}=\left(x_{2}, \ldots, x_{n}\right)$. Let $P_{j}^{0}$ be the highest 
homogeneous term of $P_{j}$. By induction one can prove that $P_{2 j}^{0}=(-1)^{j} k_{1}^{2 j} P_{0}^{0} a_{j}$ and $P_{2 j-1}^{0}=(-1)^{j-p} k_{1}^{2(j-p-1)} P_{0}^{0} c_{2 p-1} b_{j}$, where the constant $a_{j}>0$ and $b_{1}=b_{2}=\ldots=b_{p}=0$ (by assumption) and $b_{j}>0$ for $m \geq j \geq p+1$. Indeed, for $P_{2 j}^{0}$ it follows easily from the relations (23). For $P_{2 j-1}^{0}$ one can use induction arguments similar to [14] ( prop. 3.3, p. 196).

Now let's consider the equation (23) with the resonance value $j=2 m-1$ :

$$
0=\left(\widetilde{\Delta}-k^{2}\right) \psi_{2 m-1}-\sum_{i=-1}^{2 m-1} c_{i} \psi_{2 m-1-i} .
$$

Since this holds identically for all $k$ the highest homogeneous term should vanish. Simple calculation shows that this term is equal to

$$
-\left(P_{2 m-1}^{0} k_{1}^{2}+P_{2 m-2 p}^{0} c_{2 p-1}\right)=(-1)^{m-p+1} k_{1}^{2(m-p)} P_{0}^{0}\left(b_{m}+a_{m}\right) c_{2 p-1} .
$$

Since $b_{m}+a_{m}>0$ and $P_{0}^{0} \neq 0$ it vanishes only if $c_{2 p-1}=0$. This completes the proof.

It is remarkable that the BA function turns out to be symmetric with respect to $k$ and $x$. For Coxeter configurations this property has been established in [5].

Theorem 2.3. Baker-Akhiezer function $\psi(k, x)$ is symmetric with respect to $x$ and $k: \psi(k, x)=\psi(x, k)$.

Proof. The idea is to show that $\psi(x, k)$ is also the BA function and then to use the uniqueness (theorem 1.1). Let's prove that $\frac{A(x) P(k, x)}{A(k)}$ is a polynomial in $x$ with the highest term $A(x)$, where $A(x)$ and $P(k, x)$ are the same as in (7). For that let us consider the conditions (8) for $\psi(k, x)$. They give a linear system for the coefficients of the polynomial $P$ with the coefficients, which are proportional to the degrees $(\alpha, x)$, $\alpha \in \mathcal{A}$. Since this system has a unique solution, these coefficients are rational in $x$. Let' denote by $P_{j}(k, x)$ the homogeneous term of $\frac{P(k, x)}{A(k)}$ of degree $-j$ in $k$. In terms of $P_{j}(k, x)$ one can rewrite the equation (9) in the following recurrent way

$$
L P_{j}(k, x)=2 \sum_{i=1}^{n} k_{i} \frac{\partial}{\partial x_{i}} P_{j+1}, \quad P_{0}(k, x)=1 .
$$

From this it follows by induction that all the singularities of $\psi(k, x)$ in $x$ belong to our configuration of the hyperplanes $(\alpha, x)=0$. Analyzing Laurent expansions for $u(x)$ and $\psi(k, x)$ on these hyperplanes we conclude that $\psi(k, x)$ has a pole of order $m_{\alpha}$ along the hyperplanes $(\alpha, x)=0$. All that means that $A(x) P(k, x)$ is a polynomial in $x$. But from the uniqueness of BA-function it follows easily that $P_{j}(k, x)$ is also homogeneous in $x$ with the same degree $-j$. Hence the highest term in $x$ of the polynomial $A(x) P(k, x)$ is equal to $A(x) A(k)$. Thus $\psi(x, k)=\frac{A(x)+\ldots}{A(x)} e^{(k, x)}$. Properties of the Laurent expansions in $x$ follow immediately from the theorems 2.1, 2.2. So we have all the conditions for $\psi(x, k)$ to be a BA function. The theorem is proved.

Corollary 2.4. Baker-Akhiezer function $\psi$ satisfies the following bispectral problem

$$
L\left(x, \frac{\partial}{\partial x}\right) \psi(k, x)=-k^{2} \psi(k, x), \quad L\left(k, \frac{\partial}{\partial k}\right) \psi(k, x)=-x^{2} \psi(k, x)
$$

where $L$ is the Schrödinger operator (10). 
Now we are able to prove Theorem 1.3.

Corollary 2.5. The Baker-Akhiezer function $\psi$ is an eigenfunction of the operator (12) for any $f \in \mathcal{R}_{\mathcal{A}}$.

Proof. Due to the Theorem 1.2 and to the symmetry of $\psi$ for any $f \in \mathcal{R}_{\mathcal{A}}$ there exists a differential operator $A\left(k, \frac{\partial}{\partial k}\right)$ such that $A\left(k, \frac{\partial}{\partial k}\right) \psi=f(x) \psi$. On the other hand, $L\left(x, \frac{\partial}{\partial x}\right) \psi=-k^{2} \psi$. Now we can use the identity (1.8) from [14] which states in that case that

$$
(a d L)^{r}(\hat{f})[\psi]=\left(-a d \hat{k}^{2}\right)^{r}(A)[\psi]
$$

for all $r \in \mathbf{Z}_{+}$. For $r=N=\operatorname{ord} A=\operatorname{deg} f$ the differential operator $\left(-a d \hat{k}^{2}\right)^{r}(A)$ in the right-hand side has zero order and is, in fact, the operator of multiplication by $c f(k)$ with $c=(-2)^{N} N$ !. This means that $\psi$ is an eigenfunction of the operator $(a d L)^{r}(\hat{f})$ with the eigenvalue $c f(k)$. This proves the theorem 1.3.

Now let's explain why the existence of $\phi$ with the properties (13), (14) (our old axiomatics, see section 1) implies the existence of BA function $\psi$. This follows from the following general statement, showing that the new axiomatics is in some sense the most general one.

Let $\mathcal{A}$ be any configuration of hyperplanes, $L=-\Delta+u(x)$ be a corresponding Schrödinger operator, $A(k)=\prod_{\alpha \in \mathcal{A}}(\alpha, k)^{m_{\alpha}}$. Consider the functions $\varphi$ of the form

$$
\varphi(k, x)=\frac{P(k, x)}{A(k) A(x)} e^{(k, x)},
$$

$P$ is some polynomial in $k$ and $x: P=A(k) A(x)+\ldots$, where dots mean the terms of lower order both in $k$ and in $x$.

Theorem 2.6. If the Schrödinger equation $L \varphi=-k^{2} \varphi$ has a solution $\varphi$ of the form (25) then $\varphi(k, x)$ has to be BA function.

Proof now is almost evident. Theorems 2.1 and 2.2 provide the conditions (8) for $\varphi$ in $x$-variable, and it has the required form (7) in $x$. Hence, $\varphi(x, k)$ is BA function and according to the theorem $2.3 \varphi(x, k)=\varphi(k, x)$.

Corollary 2.7. If a function $\phi$ satisfies the conditions (13)-(14) then $\psi=A^{-1}(k) \phi$ is the Baker-Akhiezer function (7)-(8).

Proof. As it follows from the results of the papers [4], [5], the function $\phi$ must be an eigenfunction of the same equation (9). Then the arguments we used in the proof of the theorem 2.3 show that $\varphi=A^{-1}(k) \phi$ satisfies the conditions of the theorem 2.6 and therefore is the Baker-Akhiezer function.

\section{Locus equations and the existence of BA function.}

Let $\mathcal{A}$, as in Section 1 , be a finite set of non-collinear vectors $\alpha \in \mathbf{C}^{n}$ with given multiplicities $m_{\alpha} \in \mathbf{Z}_{+}, \mathfrak{A}$ be the corresponding configurations of hyperplanes $(\alpha, k)=0$ in $\mathbf{C}^{n}$ and $L=-\Delta+u(x)$ be the Schrödinger operator with the potential

$$
u(x)=\sum_{\alpha \in \mathcal{A}} \frac{m_{\alpha}\left(m_{\alpha}+1\right)(\alpha, \alpha)}{(\alpha, x)^{2}} .
$$

The theorems 2.1 and 2.2 from the previous section imply that if the BA function for the configuration $\mathfrak{A}$ exists then in the normal Laurent expansions (16) of the potential 
$u(x)$ the first odd terms $c_{2 j-1}^{(\alpha)}\left(j=1, \ldots, m_{\alpha}\right)$ should vanish identically on the hyperplane $(\alpha, x)=0$. More explicitly, these conditions have the form of the following highly overdetermined algebraic system:

$$
\sum_{\substack{\beta \in \mathcal{A} \\ \beta \neq \alpha}} \frac{m_{\beta}\left(m_{\beta}+1\right)(\beta, \beta)(\alpha, \beta)^{2 j-1}}{(\beta, x)^{2 j+1}} \equiv 0 \text { on the hyperplane }(\alpha, x)=0
$$

for $j=1,2, \ldots, m_{\alpha}$.

We will call the equations (27) as locus equations, following Airault, McKean and Moser [13], who used this terminology in one-dimensional case. The configurations $\mathfrak{A}$ which satisfy the locus equations we will call as locus configurations.

The remarkable fact is that the locus equations (27) are not only necessary, but are also sufficient for the existence of the BA function. We will give the proof following the paper [21].

Theorem 3.1. For any locus configuration $\mathfrak{A}$ the $B A$ function $\psi(k, x)$ does exist and can be given by the following Berest's formula:

$$
\psi(k, x)=\left[(-2)^{M} M ! A(k)\right]^{-1}\left(L+k^{2}\right)^{M}\left[\prod_{\alpha \in \mathcal{A}}(\alpha, x)^{m_{\alpha}} \exp (k, x)\right],
$$

where $M=\sum_{\alpha \in \mathcal{A}} m_{\alpha}, A(k)=\prod_{\alpha \in \mathcal{A}}(\alpha, k)^{m_{\alpha}}$.

Proof. Let's consider the linear space $V$ which consists of the functions $\phi(x), x \in$ $\mathbf{C}^{\mathbf{n}}$, with the following analytic properties:

1) $\phi(x) \prod_{\alpha \in \mathcal{A}}(\alpha, x)^{m_{\alpha}}$ is holomorphic in $\mathbf{C}^{\mathbf{n}}$;

2) for each $\alpha \in \mathcal{A}$ the Laurent expansion (15) for $\phi$ should not contain the terms of order $-m_{\alpha}+2 j-1\left(j=1, \ldots, m_{\alpha}\right)$, i.e. the conditions (20) hold.

The basic observation is the following

Lemma. The space $V$ defined above is invariant under the Schrödinger operator with the potential (26) provided that the locus conditions (27) are fulfilled.

It follows easily from the imposed conditions on the Laurent expansions in $\alpha$-direction for $u(x)$ and $\phi \in V$.

Now let's define the functions $\varphi_{i}(i=0,1, \ldots)$ in the following way:

$$
\varphi_{0}=\prod_{\alpha \in \mathcal{A}}(\alpha, x)^{m_{\alpha}} \exp (k, x)
$$

and

$$
\varphi_{i+1}=\left(L+k^{2}\right) \varphi_{i}
$$

It's obvious that $\varphi_{0}$ belongs to $V$, hence by the lemma $\varphi_{i}$ also belongs to $V$. From the definition of these functions and the property 1 of $V$ it is clear that $\varphi_{i}$ can be presented in the form $\varphi_{i}=R_{i}(k, x) \exp (k, x)$, where $R_{i}=Q_{i} \prod_{\alpha \in \mathcal{A}}(\alpha, x)^{-m_{\alpha}}$ for some polynomial $Q_{i}(k, x)$. From (29) it follows that the degrees of the polynomials $Q_{i}$ in $x$ decrease: $\operatorname{deg} Q_{i+1}<\operatorname{deg} Q_{i}$. Therefore, for some $N \quad \varphi_{N} \neq 0$ but $\varphi_{N+1}=\left(L+k^{2}\right) \varphi_{N}=0$. Thus, $\phi=\varphi_{N}$ is an eigenfunction for the Schrödinger operator $L$. Let's prove that $N$ in fact 
equals to $M=\sum_{\alpha \in \mathcal{A}} m_{\alpha}$. If we denote by $R_{i}^{0}$ the highest homogeneous terms of $R_{i}$ in $x$, we see from (29) that

$$
R_{i+1}^{0}=-2 \sum_{j=1}^{n} k_{j} \partial / \partial x_{j}\left(R_{i}^{0}\right) .
$$

From this we obtain immediately that for $i=M=\sum_{\alpha \in \mathcal{A}} m_{\alpha}$

$$
R_{M}^{0}=(-2)^{M} M ! \prod_{\alpha \in \mathcal{A}}(\alpha, k)^{m_{\alpha}}
$$

From this we conclude that for $i>M R_{i}$ (which is polynomial in $k$ ) will be of the negative degree in $x$. Thus, it cannot be an eigenfunction for the Schrödinger operator $L$ because of the following lemma due to F.A.Berezin [22].

Lemma. If a quasipolynomial $\psi$ in $k \psi=P(k, x) \exp (k, x)$ satisfies the Schrödinger equation $(-\Delta+u(x)) \psi=-k^{2} \psi$ then the highest term in $k$ of the polynomial $P$ must be polynomial in $x$.

This contradiction proves that the last non-zero function in the sequence (29) is $\phi_{M}$. Moreover, since $\phi_{M}$ belongs to the space $V$ we obtain using (30) that $\psi(k, x)=$ $\left(R_{M}^{0}\right)^{-1} \varphi_{M}$ satisfies axiomatics (7),(8) in $x$ as well as in $k$ according to the theorem 2.3. So, we proved that $\psi(k, x)$ defined by the formula (28) is the BA function associated to a configuration $\mathcal{A}$.

Remark. The remarkable formula (28) for $\psi$ was discovered by Yu. Berest ([18]), who proved that if $\psi$ does exist then it should have the form (28).

\section{Analysis of the locus equations and locus configu- rations.}

The next step would be to classify all the solutions of the locus equations (locus configurations). Unfortunately, this problem seems to be very difficult. In this section we present some results in this direction and all the known examples.

\subsection{Coxeter systems.}

The most natural examples of the locus configurations are given by the mirrors of the Coxeter groups. Recall that a Coxeter group $W$ is by definition a finite group generated by some orthogonal reflections $s_{\alpha}(x)=x-\frac{2(\alpha, x)}{(\alpha, \alpha)} \alpha$ with respect to hyperplanes in $\mathbf{R}^{n}$ (see [23]). If we consider all the reflections from the Coxeter group $W$, then the set $\mathfrak{A}$ of the corresponding hyperplanes $(\alpha, x)=0$ will be invariant under the action of $W$. The configuration $\mathfrak{A}$ of these hyperplanes with arbitrary $W$-invariant multiplicities $m_{\alpha} \in \mathbf{Z}_{+}$ gives an example of locus configuration. This fact follows immediately from the symmetry of the corresponding potential $u(x)$ with respect to any reflection $s_{\alpha}, \alpha \in \mathcal{A}$.

In this case the Schrödinger operator $L$ is the quantum Hamiltonian of the generalised Calogero - Moser system (see [24], [6]). The existence of the BA function for the root system of type $A_{n}$ with $m_{\alpha}=1$ was proved in [4], where some explicit formula for $\psi$ has been found. For the general Coxeter system it was done in [5], using the Heckman's formula [7] for the so-called shift operators in terms of the Dunkl operators [25]. Notice that our approach gives a new proof of this result. 
Remark. In principle, one may try to extend these examples to the complex case, by considering a finite group generated by orthogonal reflections in complex Euclidean space. However, it is known (see e.g. [26]) that all such groups are nothing but the complexified Coxeter groups.

\subsection{Deformed root systems.}

The first non-Coxeter locus configuration $A_{n}(m)$ was introduced in [8]. It consists of the following vectors in $\mathbf{R}^{n+1}: e_{i}-e_{j}$ with multiplicity $m(1 \leq i<j \leq n)$ and $e_{i}-\sqrt{m} e_{n+1}$ with multiplicity $1(i=1, \ldots, n)$. Notice that for $m=1$ we have the root system $A_{n}$. We can allow the parameter $m$ to be negative simply considering the vectors $e_{i}-e_{j}$ with the multiplicity $-1-m$ in that case (then, of course, we will have a complex configuration in $\mathbf{C}^{n+1}$ ).

Corresponding Schrödinger operator has the form:

$$
L=-\Delta+\sum_{i<j}^{n} \frac{2 m(m+1)}{\left(x_{i}-x_{j}\right)^{2}}+\sum_{i=1}^{n} \frac{2(m+1)}{\left(x_{i}-\sqrt{m} x_{n+1}\right)^{2}}
$$

In the simplest nontrivial case $n=2$ we have the following configuration (see figure 1).

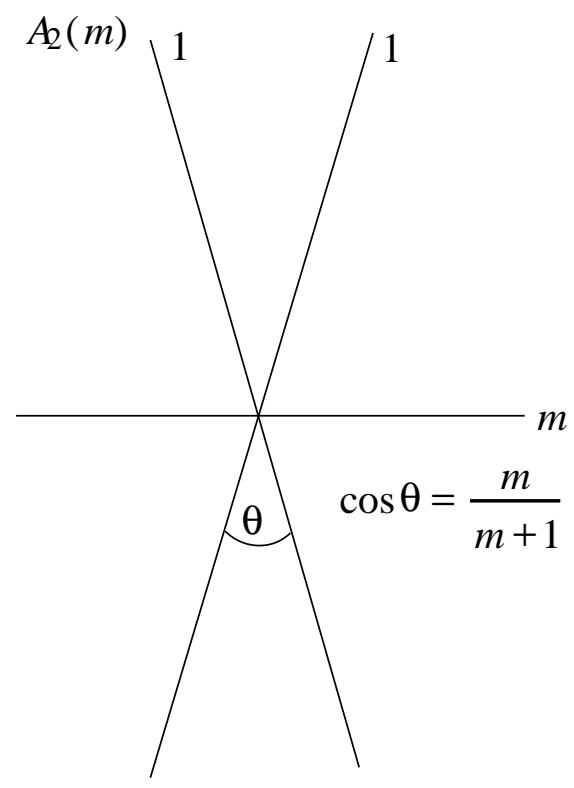

Figure 1

The next example is related to the root system of $C_{n}$ - type. Let's consider the following set of vectors in $\mathbf{R}^{n+1}$ :

$$
C_{n+1}(m, l)= \begin{cases}e_{i} \pm e_{j} & \text { with multiplicity } k \\ 2 e_{i} & \text { with multiplicity } m \\ 2 \sqrt{k} e_{n+1} & \text { with multiplicity } l \\ e_{i} \pm \sqrt{k} e_{n+1} & \text { with multiplicity } 1\end{cases}
$$


where $l$ and $m$ are integer parameters such that $k=\frac{2 m+1}{2 l+1} \in \mathbf{Z}, 1 \leq i<j \leq n$. In the case of $C_{2}(m, l)$ - system the parameters $m, l$ can be arbitrary integers, the corresponding quantum problem was considered in $[8,9]$. The corresponding configuration has the form shown on the figure 2 .

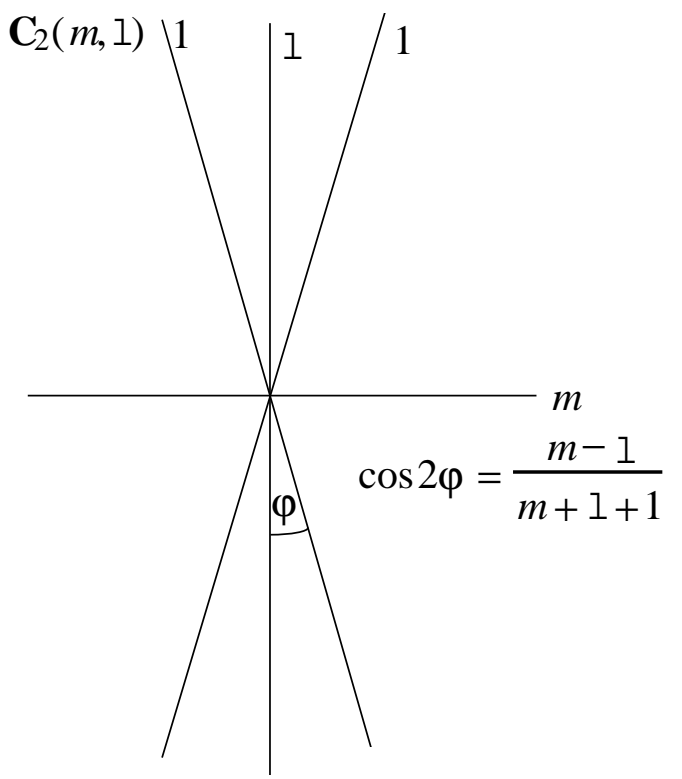

Figure 2

For $n>1$ the corresponding Schrödinger operator has the form:

$$
\begin{aligned}
L= & -\Delta_{n+1}+\sum_{i<j}^{n} \frac{4 k(k+1)\left(x_{i}^{2}+x_{j}^{2}\right)}{\left(x_{i}^{2}-x_{j}^{2}\right)^{2}}+\sum_{i=1}^{n} \frac{m(m+1)}{x_{i}^{2}}+ \\
& \frac{l(l+1)}{x_{n+1}^{2}}+\sum_{i=1}^{n} \frac{4(k+1)\left(x_{i}^{2}+k x_{n+1}^{2}\right)}{\left(x_{i}^{2}-k x_{n+1}^{2}\right)^{2}}
\end{aligned}
$$

where $k=\frac{2 m+1}{2 l+1}$. In the case $l=m$ the system $C_{n+1}(m, l)$ coincides with the classical root system $C_{n+1}$ (or $D_{n+1}$ for $l=m=0$ ). Again, as for the $A_{n}(m)$ system, the parameters $k, l, m$ may be negative, in that case the corresponding multiplicities in () should be $-1-k,-1-m$ or $-1-l$ respectively.

The simplest way to check the validity of the locus equations for these configurations is to use the following important property of the system (27):

Theorem 4.1. A configuration $\mathfrak{A}$ satisfies locus equations (27) if and only if each two-dimensional subsystem of $\mathcal{A}$ gives a locus configuration. In other words, for each two-dimensional plane $\pi \subset \mathbf{C}^{n}$ the vectors $\alpha \in \mathcal{A} \cap \pi$ with their multiplicities $m_{\alpha}$ must satisfy the locus equations.

Remark. Notice the analogy with the similar property of the Coxeter and root systems.

Proof. Let us denote by $\widehat{\beta}$ the orthogonal projection of a vector $\beta$ onto the hyperplane $(\alpha, x)=0$, then $(\widehat{\beta}, x) \equiv(\beta, x)$ on this hyperplane. Let $\pi\langle\alpha, \gamma\rangle$ denote the two-dimensional plane spanned by $\alpha$ and $\gamma \neq \alpha$. Then the subsum of (27) over $\beta \in \pi\langle\alpha, \gamma\rangle$ becomes proportional to $(\widehat{\gamma}, x)^{-2 j-1}$ being restricted to the hyperplane 
$(\alpha, x)=0$. All these subsums for different two-dimensional hyperplanes are independent, so we come to the following equivalent form of (27):

for any two-dimensional plane $\pi \in \mathbf{C}^{n}$ and for each $\alpha \in \mathcal{A} \cap \pi$ and $j=1, \ldots, m_{\alpha}$

$$
\sum_{\substack{\beta \in \mathcal{A} \cap \pi \\ \beta \neq \alpha}} m_{\beta}\left(m_{\beta}+1\right)(\beta, \beta)(\alpha, \beta)^{2 j-1}(\beta, x)^{-2 j-1} \equiv 0 \text { for }(\alpha, x)=0 .
$$

That gives the statement of the theorem.

If we analyse the configurations $A_{n}(m)$ and $C_{n+1}(m, l)$ from this point of view, we will have in each two-dimensional plane either a usual root system or one of their deformations $A_{2}(m)$ and $C_{2}(m, l)$. For these two cases the locus equations can be checked by direct calculation.

One can see that our configurations $A_{n}(m)$ and $C_{n+1}(m, l)$ have one common feature: they are obtained from Coxeter configurations by adding a special orbit of the Coxeter group with multiplicity 1 (a sort of "one-orbit deformation" of a Coxeter configuration). The following result demonstrates that such property is not accidental: the hyperplanes with large multiplicities always form a Coxeter subsystem.

Definition. Let's say that the hyperplane $\Pi_{\beta} \in \mathfrak{A}$ has a large multiplicity $m_{\beta}$ if in each two-dimensional plane containing vector $\beta$ there are no more than $m_{\beta}+1$ vectors from $\mathcal{A}$ (without taking into account the multiplicities).

Theorem 4.2. The set $\mathbf{B} \subset \mathfrak{A}$ of all hyperplanes with large multiplicities forms a Coxeter configuration and all other hyperplanes and their multiplicities are invariant under the action of this Coxeter group.

Proof. We shall prove that for each $\beta \in \mathcal{B}$ the corresponding reflection $s_{\beta}$ preserves the set $\mathfrak{A}$ together with multiplicities. This implies, in particular, that $s_{\beta}(\mathbf{B}) \subset \mathbf{B}$. To prove the invariance of $\mathfrak{A}$ under $s_{\beta}$ let's consider as in theorem 4.1 an arbitrary twodimensional plane $\pi$, which contains $\beta$, and the corresponding two-dimensional locus equation (33):

$$
\left.\sum_{\substack{\gamma \in \mathcal{A} \cap \pi \\ \gamma \neq \beta}} m_{\gamma}\left(m_{\gamma}+1\right)(\gamma, \gamma)(\beta, \gamma)^{2 j-1}(\gamma, x)^{-2 j-1}\right|_{(\beta, x)=0} \equiv 0
$$

where $j=1, \ldots, m_{\beta}$. Now we look at these equations for generic fixed $x$ as a linear system for unknowns $z_{\gamma}=m_{\gamma}\left(m_{\gamma}+1\right)(\gamma, \gamma)(\beta, \gamma)(\gamma, x)^{-3}$ of the form

$$
\left.\sum_{\gamma \neq \beta} z_{\gamma}\left(\frac{(\beta, \gamma)^{2}}{(\gamma, x)^{2}}\right)^{j-1}\right|_{(\beta, x)=0} \equiv 0, \quad j=1, \ldots, m_{\beta} .
$$

We need the following elementary lemma:

Lemma. If three unit vectors $\beta, \gamma, \gamma^{\prime}$ belong to some two-dimensional subspace in $\mathbf{C}^{n}$ and

$$
\frac{(\beta, \gamma)^{2}}{(\gamma, x)^{2}}=\frac{\left(\beta, \gamma^{\prime}\right)^{2}}{\left(\gamma^{\prime}, x\right)^{2}}
$$

for all $x$ such that $(\beta, x)=0$ then either $\gamma= \pm \gamma^{\prime}$ or $s_{\beta}(\gamma)= \pm \gamma^{\prime}$.

Let's regroup the terms in (34) into the groups corresponding to different values of $\frac{(\beta, \gamma)^{2}}{(\gamma, x)^{2}}$. From the properties of the Vandermond determinant we easily conclude that the 
sum of $z_{\gamma}$ in each group should vanish. On the other hand, using lemma we see that there are only two terms in each group, and they correspond to the pairs of vectors $\gamma, \gamma^{\prime}$ with $s_{\beta}(\gamma)= \pm \gamma^{\prime}$. Finally, we arrive to the condition $z_{\gamma}+\left.z_{\gamma^{\prime}}\right|_{(\beta, x)=0}=0$, which gives $m_{\gamma}\left(m_{\gamma}+1\right)=m_{\gamma^{\prime}}\left(m_{\gamma^{\prime}}+1\right)$, i.e. $m_{\gamma}=m_{\gamma^{\prime}}$.

Remark. The Schrödinger operators (31), (32) remain integrable in a usual (Liouville) sense for the general (non-integer) values of the parameters $m, l$ : there exists at least $n=\operatorname{dim} V$ independent commuting operators $L_{1}=L, L_{2}, \ldots, L_{n}$. Indeed, for $A_{n}(m)$ case ( $m$ is integer) it's easy to check that the polynomials $p_{s}=k_{1}^{s}+k_{2}^{s}+\ldots+k_{n}^{s}+$ $m^{\frac{s-2}{2}} k_{n+1}^{s}(s=1,2, \ldots)$ satisfy the conditions (11) and, according to the theorem 1.2, there exist differential operators $L_{s}$ with the highest symbols $p_{s}$ such that $L_{s} \psi=p_{s} \psi$ and therefore $\left[L_{s}, L_{t}\right]=0$ (see the explicit formula (12)). Since the coefficients of these operators depend on $\sqrt{m}$ in a rational way, one can define such operators for general $m$. For $s=2$ one has the Schrödinger operator (31), and other $L_{s}$ give its quantum integrals. In the case of $C_{n+1}(m, l)$ - system the similar arguments prove the integrability of the Schrödinger operator (32) for the general $l, m$, and the commuting quantum integrals $L_{s}$ have the symbols $p_{s}=k_{1}^{2 s}+\ldots+k_{n}^{2 s}+q^{s-1} k_{n+1}^{2 s}\left(q=\frac{2 m+1}{2 l+1}, s=1,2, \ldots\right)$.

\subsection{Locus configurations on the plane.}

Yu.Berest and I.Lutsenko [11] in the context of the Huygens' Principle have introduced the following family of the real potentials $u$ on the real plane. In the polar coordinates they have a form

$$
u(r, \varphi)=-\frac{2}{r^{2}} \frac{\partial^{2}}{\partial \varphi^{2}} \log W\left[\chi_{1}(\varphi), \ldots, \chi_{M}(\varphi)\right]
$$

where $\chi_{j}(\varphi)=\cos \left(k_{j} \varphi+\theta_{j}\right), k_{M}>\ldots>k_{1}>0, k_{j} \in \mathbf{N}, \theta_{j} \in \mathbf{R}$ and $W\left[\chi_{1}, \ldots, \chi_{M}\right]$ is the Wronskian of $\chi_{1}, \ldots, \chi_{M}$.

One can consider the natural complexification of the Berest-Lutsenko family in the following way. The set of all non-isotropic lines in $\mathbf{C}^{2}$ is isomorphic to the cylinder $\mathbf{C}^{*} \simeq \mathbf{C} P^{1} \backslash\{0, \infty\}$ and can be parametrised by a complex parameter $\varphi(\bmod \pi)$

$$
x \cos \varphi+y \sin \varphi=0 .
$$

Any configuration corresponds to a finite number of points in $\mathbf{C}^{*}: \varphi_{1}, \ldots, \varphi_{N}$ with multiplicities $m_{1}, \ldots, m_{N}$. The corresponding potential has the form

$$
u=\frac{1}{r^{2}} \sum_{j=1}^{N} \frac{m_{j}\left(m_{j}+1\right)}{\sin ^{2}\left(\varphi-\varphi_{j}\right)}
$$

where $r^{2}=x^{2}+y^{2} \in \mathbf{C} \backslash\{0\}$ and $\varphi(\bmod \pi)=\arctan \frac{y}{x}$. The complex Berest-Lutsenko potentials given by the formula (35) with the complex parameters $\theta_{j}$, have the form (36) with $\varphi_{j}$ being the roots of the trigonometric polynomial $W[\varphi]$, their multiplicities are known to have a "triangular" form $\frac{m_{j}\left(m_{j}+1\right)}{2}$ (see [13]).

Theorem 4.3. All the locus configurations on the plane are determined by the complex Berest-Lutsenko formula (35).

Proof. First of all the locus equations (27) in this case are equivalent to the following one-dimensional locus equations (cf. [13]) for the potential $v(\varphi)=\sum_{j=1}^{N} \frac{m_{j}\left(m_{j}+1\right)}{\sin ^{2}\left(\varphi-\varphi_{j}\right)}$ :

$$
\left.\left(\frac{d}{d \varphi}\right)^{2 s-1}\left(\sum_{j \neq i} \frac{m_{j}\left(m_{j}+1\right)}{\sin ^{2}\left(\varphi-\varphi_{j}\right)}\right)\right|_{\varphi=\varphi_{i}}=0 \quad\left(i=1, \ldots, N, s=1,2, \ldots, m_{i}\right) .
$$


Now we can use the result from [21], which says that in its turn this is equivalent to the existence of the differential operator $D$ with $\pi$-periodic coefficients, intertwining the operator $\mathcal{L}=-\frac{d^{2}}{d \varphi^{2}}+v(\varphi)$ with $\mathcal{L}_{0}=-\frac{d^{2}}{d \varphi^{2}}$ :

$$
\mathcal{L} \circ D=D \circ \mathcal{L}_{0}
$$

The idea of the proof is close to the one demonstrated in the proof of the theorem 3.1, and we shall not reproduce it here.

So, the only remaining thing to prove is that the relation (37) implies that $\mathcal{L}$ can be obtained from $\mathcal{L}_{0}$ by classical Darboux transformations. Let's assume that $D$ has the minimal order among all the intertwiners of $\mathcal{L}$ and $\mathcal{L}_{0}$ and consider its kernel: $V=\operatorname{Ker} D$. As it follows from (37) $V$ is invariant under $\mathcal{L}_{0}$ : if $D f=0$ then $D\left(\mathcal{L}_{0} f\right)=$ $\mathcal{L} D f=0$. Due to $\pi$-periodicity of the coefficients of $D, \operatorname{Ker} D$ is also invariant under the shift $T: f(\varphi) \rightarrow f(\varphi+\pi)$.

We would like to show that the spectrum of $\left.\mathcal{L}_{0}\right|_{V}$ is simple and has the form $\left(k_{1}^{2}, k_{2}^{2}, \ldots, k_{M}^{2}\right)$, where $0<k_{1}<k_{2}<\ldots<k_{M}$ are some integers. Suppose that there exists an eigenfunction $f \in V$ with the eigenvalue $\lambda \neq k^{2}, k \in \mathbf{Z}$. Since $\mathcal{L}_{0}$ commutes with $T$, we can assume that $f$ is a Bloch eigenfunction:

$$
\left\{\begin{aligned}
\mathcal{L}_{0} f & =\lambda f \\
T f & =\mu f
\end{aligned}\right.
$$

If $\lambda \neq k^{2} \quad f$ has to be pure exponent: $f=C e^{\sqrt{-\lambda} \varphi}$ or $f=C e^{-\sqrt{-\lambda} \varphi}$. Since $D f=0$ the operator $D$ can be factorised as

$$
D=\widetilde{D} \circ F, \quad F=\frac{d}{d \varphi}-\frac{f^{\prime}}{f}
$$

where $\widetilde{D}$ is a $\pi$-periodic differential operator of the order one less than $D$ (see e.g. [27]). When $f=C e^{ \pm \sqrt{-\lambda} \varphi}$ we have $F=\frac{d}{d \varphi} \pm \sqrt{-\lambda}$ and $\mathcal{L} \circ \widetilde{D} \circ F=\widetilde{D} \circ F \circ \mathcal{L}_{0}=\widetilde{D} \circ \mathcal{L}_{0} \circ F$. Thus $\mathcal{L} \circ \widetilde{D}=\widetilde{D} \circ \mathcal{L}_{0}$, so $\widetilde{D}$ is also an intertwiner with the order one less than the order of $D$.

Thus the spectrum of $\left.\mathcal{L}_{0}\right|_{V}$ consists only of the squares of integers: $\lambda=k^{2}, k \in \mathbf{Z}$. The same arguments show that $\lambda \neq 0$. So we have only to prove that the spectrum is simple. First of all there could be only one eigenfunction, corresponding to a given $\lambda=k^{2}$. Indeed, otherwise $\operatorname{Ker} D$ contain the whole $\operatorname{Ker}\left(\mathcal{L}_{0}-\lambda\right)$ and therefore $D$ can be factorised $D=D_{1} \circ\left(\mathcal{L}_{0}-\lambda\right)$ with $D_{1}$ being another intertwiner of less order. Suppose that $\mathcal{L}_{0}$ has a Jordan block with $\lambda=k^{2}$. Consider the Jordan basis $f_{0}, f_{1}, \ldots$ : $\left(\mathcal{L}_{0}-\lambda\right) f_{0}=0,\left(\mathcal{L}_{0}-\lambda\right) f_{1}=f_{0}, \ldots$. Since $f_{0}$ can not be pure exponent (see above), $f_{0}=A \cos \left(k \varphi+\theta_{0}\right)$, then $f_{1}=\frac{A \varphi}{2 k} \sin \left(k \varphi+\theta_{0}\right)+B \cos \left(k \varphi+\theta_{1}\right)$. Now from the invariance of $\operatorname{KerD}$ under the shift $T$ we conclude that $\frac{A \pi}{2 k} \sin \left(k \varphi+\theta_{0}\right)$ also belongs to $\operatorname{Ker} D$. Together with $f_{0}$ the last function generates $\operatorname{Ker}\left(\mathcal{L}_{0}-\lambda\right)$, which leads to factorisation $D=D_{1} \circ\left(\mathcal{L}_{0}-\lambda\right)$ and reducibility of $D$.

Thus we have proven that $\operatorname{Ker} D$ is generated by the functions $\chi_{1}, \ldots, \chi_{n}$ of the form $\chi_{j}=\cos \left(k_{j} \varphi+\theta_{j}\right)$. The general formula (see e.g. [29]) from the theory of Darboux transformations says that $u=-2 \frac{d^{2}}{d \varphi^{2}} \log W\left[\chi_{1}, \ldots, \chi_{n}\right]$. The theorem is proven. 
We should mention that although the formula (35) is explicit, it is not so easy to extract the geometric information about the locus configurations. For example, it is not clear how to prove the following theorem using this formula.

It is very easy to show that all two-line locus configurations consist of two perpendicular lines with arbitrary multiplicities. Let's consider the first non-trivial case of three lines $(\alpha, x)=0,(\beta, x)=0$ and $(\gamma, x)=0, x \in \mathbf{C}^{2}$ with arbitrary multiplicities $m_{\alpha}, m_{\beta}, m_{\gamma} \in \mathbf{Z}_{+}$, and ask when they form a locus configuration. Modulo the natural rotational equivalence we have the following classification.

Theorem 4.4. All the three lines locus configurations are listed below:

1) the Coxeter $A_{2}$ configuration with multiplicities $(m, m, m)$;

2) the deformed $A_{2}(m)$ configuration (31) with multiplicities $(1,1, m)$ when $m$ is positive and $(1,1,-m-1)$ when $m$ is negative;

3) the three lines complex Berest-Lutsenko configurations, which can be parametrised in this case as:

$$
\alpha=(1, a), \beta=(1, b), \gamma=(0,1): a^{2}-a b+b^{2}+1=0,
$$

the multiplicities are $(1,1,1)$.

Proof. Let $\mathfrak{A}$ be an arbitrary three lines locus configuration. Let us consider the first case when $\mathfrak{A}$ has at least two vectors with multiplicities greater than 1 . Then the theorem 4.2 states that $\mathfrak{A}$ has to be a Coxeter $A_{2}$ - system. Now let us suppose that there is only one vector $\gamma=(0,1)$ with multiplicity $m>1$. The theorem 4.2 states that other two vectors have to be symmetric with respect to the vector $\gamma$, so we may fix the normalisation $\alpha=(1, \lambda), \beta=(1,-\lambda)$. The locus equation (27) for $\alpha$ has a form:

$$
\frac{2\left(1+\lambda^{2}\right)\left(1-\lambda^{2}\right)}{(x-\lambda y)^{3}}+\frac{m(m+1) \lambda}{y^{3}}=0 \quad \text { if } \quad x+\lambda y=0 \text {. }
$$

From that it immediately follows that $\lambda$ can take only the following values: $\lambda=$ $\pm \frac{1}{\sqrt{2 m+1}}, \pm \frac{i}{\sqrt{2 m+1}}$, and it is easy to check that $\mathfrak{A}$ is equivalent to the system $A_{2}(m)$ or $A_{2}(-m-1)$. The last case we have to consider is the case when all the three vectors $\alpha=(1, a), \beta=(1, b), \gamma=(0,1)$ have multiplicity 1 . The locus equation (27) for vector $\gamma$ takes the form

or

$$
\frac{2 a\left(a^{2}+1\right)}{(x+a y)^{3}}+\frac{2 b\left(b^{2}+1\right)}{(x+b y)^{3}}=0 \text { if } y=0
$$

$$
(a+b)\left(a^{2}+b^{2}-a b+1\right)=0 .
$$

The locus equations (27) corresponding to $\alpha$ and $\beta$ can be written as follows:

$$
\left\{\begin{array}{l}
\left(1+a^{2}\right)(1+a b)+b(a-b)^{3}=0 \\
\left(1+b^{2}\right)(1+a b)+a(b-a)^{3}=0
\end{array}\right.
$$

In the case $a+b=0$ this system of equations is fullfilled if and only if $a^{4}=\frac{1}{9}$, which implies that $\mathfrak{A}$ is either Coxeter system $A_{2}$ or deformed system $A_{2}(-2)$. In the case $a^{2}+$ $b^{2}-a b+1=0$ the above system holds automatically without any additional restrictions. Thus, the theorem is proven.

Remark 1. We should mention that some of the configurations 3) contain an isotropic line $(a= \pm i, b=0$ or $a=0, b= \pm i)$ and therefore actually reduce to the two-line configurations. Notice also that when $a=i / \sqrt{3}=-b$ we have $A_{2}(-2)$ configuration. 
Remark 2. It can be checked that for the configurations 3) from the theorem 4.4 the function $\phi$ with the properties (13-14) doesn't exist. This demonstrates that the converse for the statement of the Corollary 2.7 is not true.

Notice that from this result it follows that the locus of $n$ lines is non-empty only for the special sets of multiplicities. Moreover, if the locus configuration is real than the set of multiplicities determines it uniquely up to rotation due to the following result.

Theorem 4.5. There exists no more than one locus configuration in $\mathbf{R}^{2}$ with given cyclically ordered set of multiplicities.

Proof. Let $\mathcal{A}=\left\{\alpha_{1}, \ldots, \alpha_{N}\right\}$ be such a configuration for given set of multiplicities $\left\{m_{1}, \ldots, m_{N}\right\}$, and let us fix normalisation $\alpha_{i}=\left(-\sin \varphi_{i}, \cos \varphi_{i}\right), 0 \leq \varphi_{1}<\varphi_{2}<\ldots<$ $\varphi_{N}<\pi$. Considering the locus equations, we have, in particular, that

$$
\sum_{\substack{j=1 \\ j \neq i}}^{N} \frac{m_{j}\left(m_{j}+1\right) \cos \left(\varphi_{j}-\varphi_{i}\right)}{\sin ^{3}\left(\varphi_{j}-\varphi_{i}\right)}=0 \text { for } i=1, \ldots, N .
$$

Let's now introduce the function

$$
U\left(\varphi_{1}, \ldots, \varphi_{N}\right)=\sum_{i<j} \frac{m_{i}\left(m_{i}+1\right) m_{j}\left(m_{j}+1\right)}{\sin ^{2}\left(\varphi_{i}-\varphi_{j}\right)} .
$$

We conclude that if $\Phi=\left(\varphi_{1}, \ldots, \varphi_{N}\right)$ defines a locus configuration then necessarily

$$
\frac{\partial}{\partial \varphi_{i}} U\left(\varphi_{1}, \ldots, \varphi_{N}\right)=0
$$

Function $U$ being a sum of convex functions is a convex function in the domain $0 \leq$ $\varphi_{1}<\varphi_{2}<\ldots<\pi$. Suppose it has one more extremum in the point $\widetilde{\Phi}=\left(\tilde{\varphi}_{1}, \ldots, \tilde{\varphi}_{N}\right)$. Then $U\left(\varphi_{1}, \ldots, \varphi_{N}\right)$ should be a constant along the segment $\Phi+(\widetilde{\Phi}-\Phi) t, 0 \leq t \leq 1$, as well as each function $\frac{m_{i}\left(m_{i}+1\right) m_{j}\left(m_{j}+1\right)}{\sin ^{2}\left(\varphi_{i}-\varphi_{j}\right)}$. From that it follows that $\tilde{\varphi}_{i}=\varphi_{i}+\varphi_{0}$ for some constant $\varphi_{0}$ for all $i$. This means that system $\left\{\alpha_{i}\right\}$ is defined uniquely up to a rotation.

Corollary 4.6. If all the multiplicities are equal then the only real configuration on the plane is Coxeter, i.e. dihedral.

The consideration of all two-dimensional subsystems implies the following more general result.

Corollary 4.7 Any real locus configurations in $\mathbf{R}^{n}$ with equal multiplicities must be Coxeter.

\section{Affine locus.}

In this section we present some results concerning the case, when the singular set of the potential $u(x)$ of the Schrödinger operator is an affine configuration $\mathfrak{S}$ of hyperplanes. So, we consider a Schrödinger operator $L=-\Delta+u(x)$ with rational potential having second order poles along some non-isotropic hyperplanes in $\mathbf{C}^{n}$. Let $\left(\alpha_{s}, x\right)+c_{s}=0$ $(s=1, \ldots, K)$ be the equations of these hyperplanes. We will suppose also that the potential $u(x)$ decays at infinity, i.e. $u(x) \rightarrow 0$ while $x \rightarrow \infty$ along the rays outside singularities. 
Impose now the condition that $L$ has local trivial monodromy around its singularities. Then the Theorem 2.1 from the section 2 allows us to reformulate this condition as some algebraic conditions on the arrangement $\mathfrak{S}$ of the singular hyperplanes $\left(\alpha_{j}, x\right)+c_{j}=0$. First of all, it follows that the potential $u(x)$ must be of the form

$$
u(x)=\sum_{j=1}^{K} \frac{m_{j}\left(m_{j}+1\right)\left(\alpha_{j}, \alpha_{j}\right)}{\left(\left(\alpha_{j}, x\right)+c_{j}\right)^{2}}
$$

for some integers $m_{1}, \ldots, m_{K}$. Then the conditions (19) imply that the Schrödinger operator with the potential of the form (38) has local trivial monodromy around its singularities if and only if the following relations are satisfied:

$$
\sum_{j \neq i} \frac{m_{j}\left(m_{j}+1\right)\left(\alpha_{j}, \alpha_{j}\right)\left(\alpha_{i}, \alpha_{j}\right)^{2 s-1}}{\left(\left(\alpha_{j}, x\right)+c_{j}\right)^{2 s+1}} \equiv 0
$$

identically on the hyperplane $\left(\alpha_{i}, x\right)+c_{i}=0$ for all $i=1, \ldots, K$ and $s=1, \ldots, m_{i}$.

We will call the relations (39) as locus equations. The equations (27) from the Section 3 are their particular case, when all the hyperplanes pass through the origin. Sometimes we will refer to (39) and (27) as to affine and linear cases correspondently.

As it follows from the section 2, the locus equations (39) are necessary for the existence of a certain eigenfunction of the corresponding Schrödinger operator $L$ (see theorem 2.2). As well as in the linear case (Section 3) the equations (39) are sufficient for this. The following result has been proven in [21].

Theorem 5.1. Let $L=-\Delta+u(x)$ be a Schrödinger operator with the potential of the form (38) which satisfies the affine locus equations (39). Then $L$ has an eigenfunction $\phi$ of the form $\phi(k, x)=P(k, x) \exp (k, x)$, where $P$ is polynomial in $k, L \phi=-k^{2} \phi$.

This eigenfunction (up to a normalization factor ) is given by the Berest's formula analoguous to (28):

$$
\psi(k, x)=\left[(-2)^{M} M ! C(k)\right]^{-1}\left(L+k^{2}\right)^{M}\left[\prod_{j=1}^{K}\left(\left(\alpha_{j}, x\right)+c_{j}\right)^{m_{j}} \exp (k, x)\right],
$$

where $M=\sum_{j=1}^{K} m_{j}$ and $C(k)=\prod_{j=1}^{K}\left(\alpha_{j}, k\right)^{m_{j}}$. The normalization is chosen in such way that $\psi(k, x)=(1+o(1)) \exp (k, x)$ as $k \rightarrow \infty$.

We start the analysis of the affine locus equations and their solutions (locus configurations) from the one-dimensional case.

\subsection{One-dimensional case.}

In this case we have a configuration of $K$ points $z_{1}, \ldots, z_{K}$ with multiplicities $m_{1}, \ldots, m_{K}$ on the complex plane and the potential

$$
u(z)=\sum_{j=1}^{K} \frac{m_{j}\left(m_{j}+1\right)}{\left(z-z_{j}\right)^{2}} .
$$

The locus equations in this case (for $m_{j}=1$ ) have been introduced in the paper by Airault, McKean and Moser [13]. Duistermaat and Grübaum [14] obtained them for the general multiplicities and proved that they are equivalent to the existence of the 
differential operator $D$ with rational coefficients, intertwining $L=-\frac{d^{2}}{d z^{2}}+u(z)$ and $L_{0}=-\frac{d^{2}}{d z^{2}}$ :

$$
L \circ D=D \circ L_{0}
$$

All such operators $L$ are the results of the classical Darboux transformations applied to $L_{0}$, so the potential $u(z)$ can be given in this case in terms of the Wronskians by the well-known explicit formula:

$$
u(z)=-2 \frac{d^{2}}{d z^{2}} \log W\left[\chi_{1}, \ldots, \chi_{m}\right]
$$

where the polynomials $\chi_{1}, \ldots, \chi_{m}$ are defined by the recurrent relations $\chi_{1}^{\prime \prime}=0, \chi_{2}^{\prime \prime}=$ $\chi_{1}, \ldots, \chi_{m}^{\prime \prime}=\chi_{m-1}$ (see Burchnall-Chaundy [32], Adler-Moser [15]). The Wronskian is a polynomial $P_{m}\left(z, c_{1}, \ldots, c_{m}\right)$ with the coefficients depending on the additional integration constants $c_{1}, \ldots, c_{m}$ (see [15] for the details).

Thus, the locus in the one-dimensional case is a union of the rational algebraic varieties of the dimensions $m=1,2,3, \ldots$, parametrised by $c_{1}, \ldots, c_{m}$, and the locus configurations are simply the roots of the corresponding Schur polynomials $P_{m}\left(z, c_{1}, \ldots, c_{m}\right)$. The solution $\psi$ of the corresponding Schrödinger equation $-\psi^{\prime \prime}+u(z) \psi=-\lambda^{2} \psi$ has a form

$$
\psi=\left(1+\sum_{i=1}^{m} a_{i}(z) \lambda^{-i}\right) e^{\lambda z} .
$$

This is a degenerate rational case of the hyperelliptic BA function, corresponding to a general finite-gap operator [2]. These rational BA functions $\psi(41)$ are characterized by the following properties in the spectral parameter (cf. [33]). Let $\xi_{1}, \ldots, \xi_{m}$ be arbitrary parameters, $\psi_{s}$ be the Laurent coefficients of $\psi$ at $\lambda=0: \psi=\sum_{s=-m}^{+\infty} \lambda^{s} \psi_{s}(z)$. Impose the following $m$ linear conditions on the coefficients $\psi_{-m}, \ldots, \psi_{m-1}$ :

$$
\left\{\begin{array}{l}
\psi_{m-1}+\sum_{s=1}^{m} \xi_{s} \psi_{m-2 s}=0 \\
\psi_{m-3}+\sum_{s=1}^{m-1} \xi_{s} \psi_{m-2 s-2}=0 \\
\psi_{m-5}+\sum_{s=1}^{m-2} \xi_{s} \psi_{m-2 s-4}=0 \\
\cdots \\
\psi_{-m+1}+\xi_{1} \psi_{-m}=0
\end{array}\right.
$$

They are equivalent to a non-degenerate system for $m$ unknown functions $a_{i}(z)$ and determine $\psi$ of the form (41) uniquely. The usual arguments [1], [33] show that such a function satisfies the Schrödinger equation $-\psi^{\prime \prime}+u(z) \psi=-\lambda^{2} \psi$ with the rational potential

$$
u(z)=2 a_{1}^{\prime}(z) .
$$

Notice that for given $\xi_{1}, \ldots, \xi_{m}$ the system (42) determines a $m$-dimensional linear subspace $V\left(\xi_{1}, \ldots, \xi_{m}\right)$ in $\mathbf{C}^{2 m}$ and therefore corresponds to a point of the Grassmannian 
$\operatorname{Gr}(m, 2 m)$. It is more convinient to identify the system of conditions (42) with a point of some infinite-dimensional Grassmannian $G r_{0}^{(2)}$ (see [33] for the details). Namely, let's consider the linear space $\mathbf{C}[[\lambda]]$ of formal series in $\lambda$, and let $W$ be a subspace of $\mathbf{C}[[\lambda]]$ with the following properties:

1) $\lambda^{m} \mathbf{C}[\lambda] \subset W \subset \lambda^{-m} \mathbf{C}[\lambda]$ where $\mathbf{C}[\lambda]$ is the space of polynomials and both inclusions have the same codimension $m$;

2) $\lambda^{2} W \subset W$.

We will suppose that the number $m=m(W)$ in 1) cannot be reduced. The set of all such subspaces for $m=0,1,2, \ldots$ we will denote as $G r_{0}^{(2)}$ following [33].

It is easy to see that the subspace of $\mathbf{C}[[\lambda]]$ consisting of all Laurent series $\psi=$ $\sum_{s=-m}^{+\infty} \lambda^{s} \psi_{s}$ which satisfy the conditions (42) represent nothing but a general point of $G r_{0}^{(2)}$. In these notations the one-dimensional BA function corresponding to $W$ is the unique element $\psi_{W}$ of the form (41) which Laurent expansion at $\lambda=0$ belongs to $W$ for each $z$. We will denote by $u_{W}$ the corresponding potential (43).

These considerations suggest the following extension of the axiomatics (7-8) of the multidimensional BA function.

\subsection{Equipped configurations and BA functions.}

Let $\mathcal{A}$ be again a finite set of non-collinear vectors in $\mathbf{C}^{n}$. We will prescribe to each vector $\alpha \in \mathcal{A}$ a subspace $W^{(\alpha)} \in G r_{0}^{(2)}$, and denote the corresponding integer $m\left(W^{(\alpha)}\right)$ as $m_{\alpha}$. We will call the corresponding set of hyperplanes $\Pi_{\alpha}:(\alpha, k)=0$ with the prescribed subspaces $W^{(\alpha)}$ as equipped configuration $\mathfrak{A}$.

Definition. For a given equipped configuration $\mathfrak{A}$ the function $\psi(k, x)$ is called the Baker-Akhiezer function if it satisfies the following two conditions:

1) $\psi$ has the form

$$
\psi=\frac{P(k, x)}{A(k)} e^{(k, x)}
$$

where $A(k)=\prod_{\alpha \in \mathcal{A}}(\alpha, k)^{m_{\alpha}}, P$ is a polynomial in $k$ with the highest term $A(k)$; 2) for each $\alpha \in \mathcal{A}$ the Laurent expansion of $\psi$ in $k$ in $\alpha$-direction calculated at any point of the hyperplane $\Pi_{\alpha}$ must belong to $W^{(\alpha)}$.

Here by the Laurent expansion of a meromorphic function $F(k)$ in $\alpha$-direction at a point $k_{0}$ we mean the Laurent expansion of the function $f(\lambda)=F\left(k_{0}+\lambda \alpha\right)$ at $\lambda=0$.

If for each subspace $W^{(\alpha)}$ the corresponding parameters $\xi$ in (42) are zeros, our definition reduces to the definition of the BA function from the Section 1. Now we will prove the analogues of the theorems 1.1, 1.2 for a general equipped configuration.

Theorem 5.2. If for a given equipped configuration $\mathfrak{A}$ there exists BA function $\psi$ then it is unique and satisfies the Schrödinger equation

$$
\left(-\Delta+\sum_{\alpha \in \mathcal{A}}(\alpha, \alpha) u_{\alpha}((\alpha, x))\right) \psi=-k^{2} \psi,
$$

where $u_{\alpha}(z)=u_{W^{(\alpha)}}(z)$ are the one-dimensional potentials, corresponding to the subspaces $W^{(\alpha)}$.

Theorem 5.3. Let $\mathcal{R}$ be the ring of polynomials $f(k)$ with the following properties:

for each $\alpha \in \mathcal{A}$ and any point $k_{0} \in \Pi_{\alpha}$ the polynomial $f_{\alpha, k_{0}}(\lambda)=f\left(k_{0}+\lambda \alpha\right)$ preserves the space $W^{(\alpha)}: f_{\alpha, k_{0}} W^{(\alpha)} \subset W^{(\alpha)}$. 
If the Baker-Akhiezer function $\psi(k, x)$ exists then for any polynomial $f(k) \in \mathcal{R}$ there exists some differential operator $L_{f}\left(x, \frac{\partial}{\partial x}\right)$ such that

$$
L_{f} \psi(k, x)=f(k) \psi(k, x) .
$$

All such operators form a commutative ring isomorphic to the ring $\mathcal{R}$. The Schrödinger operator (45) corresponds to $f(k)=-k^{2}$.

The proofs of the theorems above follow in a standard way (cf. [4]) from the following two lemmas.

Lemma 1. If some function $\psi$ of the form (44) (without the restrictions on the highest term of the polynomial $P$ ) satisfies the conditions 2 from the definition of the $B A$ function then the highest term in $P$ must be divisible by $A(k)=\prod_{\alpha \in \mathcal{A}}(\alpha, k)^{m_{\alpha}}$.

Lemma 2. The BA function corresponding to an equipped configuration $\mathfrak{A}$ has the following asymptotic behaviour at infinity:

$$
\psi(k, x)=\exp (k, x)\left(1+\sum_{\alpha \in \mathcal{A}} a_{1}^{(\alpha)}((\alpha, x)) \frac{(\alpha, \alpha)}{(\alpha, k)}+o\left(k^{-1}\right)\right)
$$

where $a_{1}^{(\alpha)}(z)$ are the first coefficients in the corresponding functions (41) $\psi_{\alpha}=\psi_{W^{(\alpha)}}$ and $o\left(k^{-1}\right)$ means the rational function of $k$ which degree is less than -1 .

To prove the lemmas, let's expand $\psi$ in Laurent series in $(\alpha, k)$ on the hyperplane $(\alpha, k)=0$. For convenience we may suppose that $(\alpha, \alpha)=1$ and choose orthonormal basis in $k$ such that $(\alpha, k)=k_{1}$, the other coordinates $k_{2}, \ldots, k_{n}$ we shall denote by $\tilde{k}$. Then up to unessential factor $\exp \left(k_{2} x_{2}+\ldots+k_{n} x_{n}\right) \quad \psi$-function (44) takes the form:

$$
\tilde{\psi}(k, x)=e^{x_{1} k_{1}} \sum_{s \geq-m_{\alpha}} k_{1}^{s} a_{s}(\tilde{k}, x),
$$

and the Laurent coefficients $a_{s}$ are rational functions of $\tilde{k}$ with possible singularities at zeros of homogeneous polynomial $\tilde{A}(\tilde{k})=\left.k_{1}^{-m} A(k)\right|_{k_{1}=0}$. Since the sum $\sum_{s \geq-m_{\alpha}} k_{1}^{s} a_{s}$ is the Laurent expansion for $\frac{P(k, x)}{A(k)}$, the degrees in $\tilde{k}$ of its coefficients $a_{s}$ decrease at $s \rightarrow \infty$ (by definition, $\operatorname{deg} \frac{p}{q}=\operatorname{deg} p-\operatorname{deg} q$ ). Now we restrict our attention to the terms $k_{1}^{s} a_{s}$ with the maximal degree of $a_{s}$ in $\tilde{k}$. From the remark above it follows that we have a finite number of such terms, and if we extract the highest homogeneous part in $\tilde{k}$ in each term, we obtain the following finite expression

$$
\tilde{\psi}^{0}\left(k_{1}, x\right)=e^{x_{1} k_{1}} \sum_{s \geq-m_{\alpha}} k_{1}^{s} a_{s}^{0}(\tilde{k}, x)
$$

where $a_{s}^{0}$ is the highest term in $a_{s}$ and all the $a_{s}^{0}$ have the same degree in $\tilde{k}$. It is clear now that constructed in that way $\tilde{\psi}^{0}$ must obey the same restrictions (42). This implies, in particular, that the sum (47) contains at least one term with $s \geq 0$. The outcome is that if we expand $P(k, x)$ in the series in $k_{1}, P=\sum_{j \geq 0} k_{1}^{j} p_{j}(\tilde{k})$, and then extract from this sum the terms with the maximal degree in $\tilde{k}$, the result must contain at least one term with $j \geq m_{\alpha}$. Now let's present $P$ as a sum of homogeneous in $k_{1}, \ldots, k_{n}$ components $P=P_{0}+P_{1}+\ldots$, and suppose that the highest term $P_{0}$ is not divisible by 
$k_{1}^{m_{\alpha}}$. In this case some other term $P_{i}$ must contain $k_{1}^{m_{\alpha}}$, but its degree in $\tilde{k}$ is clearly less than the degree of the term coming from $P_{0}$. This contradiction proves the lemma 1.

Moreover, in the extreme case when $P_{0}$ has the form $P_{0}=k_{1}^{m_{\alpha}} Q_{0}$ with $\left.Q_{0}\right|_{k_{1}=0} \neq 0$ the reduced $\psi$-function (47) up to a factor coincides with the one-dimensional BA function (41) $\psi\left(k_{1}, x_{1}\right)$. It's easy to see that this factor is simply $\left.Q_{0}\right|_{k_{1}=0}$.

In particular, this implies that the second homogeneous term $P_{1}$ in $P(k, x)$ for the BA function $\psi$ satisfies the following condition:

$$
\left[k_{1}^{1-m_{\alpha}} P_{1}\right]_{k_{1}=0}=a_{1}\left(x_{1}\right)\left[k_{1}^{-m_{\alpha}} P_{0}\right]_{k_{1}=0}
$$

where $a_{1}$ is the first coefficient in the corresponding one-dimensional BA function (41). We obtained this formula under assumption that $(\alpha, \alpha)=1$, in general it looks as follows:

$$
\left[(\alpha, k)^{1-m_{\alpha}} P_{1}\right]_{(\alpha, k)=0}=(\alpha, \alpha) a_{1}((\alpha, x))\left[(\alpha, k)^{-m_{\alpha}} P_{0}\right]_{(\alpha, k)=0} .
$$

Taking into account the restrictions (48) for all the hyperplanes $(\alpha, k)=0$, and we obtain that if $P_{0}=A(k)=\prod_{\alpha \in \mathcal{A}}(\alpha, k)^{m_{\alpha}}$, then

$$
P_{1}=A(k) \sum_{\alpha \in \mathcal{A}} a_{1}^{(\alpha)}((\alpha, x)) \frac{(\alpha, \alpha)}{(\alpha, k)},
$$

which proves the lemma 2.

Let's consider now for a given equipped configuration $\mathfrak{A}$ the corresponding Schrödinger operator (45). It is clear that the potential has the form (38). The corresponding affine configuration of the hyperplanes $\mathfrak{S}$ we will call $d u a l$ to the equipped configuration $\mathfrak{A}$. Suppose that the corresponding BA function does exist, then from the theorem 2.2 we conclude that the Schrödinger operator (45) has local trivial monodromy and hence satisfies the locus equations (39). In other words, the dual configuration $\mathfrak{S}$ must be a locus configuration. We believe that the converse is true, that is, each locus configuration appears in such way for appropriate BA function. The part 2 of the Theorem 5.6 below shows that each locus configuration is dual to some equipped configuration. So, the only problem is to check that for the function defined by the formula (40) the properties 2 from the definition of the BA function hold. Unfortunately, we couldn't find a proof for this. We can only remark that for all known affine locus configurations it is true.

\subsection{Geometry of affine locus.}

First of all, it is easy to check that the following operations preserve the locus equations and therefore allow to produce the locus configurations:

1) motions of the complex Euclidean space $\mathbf{C}^{n}$;

2) extensions of the configurations in $\mathbf{C}^{n}$ to $\mathbf{C}^{m}, m>n$, induced by an orthogonal projection $\mathbf{C}^{m} \rightarrow \mathbf{C}^{n}$

3) union of two configurations which are orthogonal to each other.

At the moment all known examples of the affine locus configurations can be constructed using these operations from one-dimensional affine and multidimensional linear locus configurations.

In particular, this is true for the configurations, corresponding to the operators introduced by Yu.Berest and P.Winternitz [16]. Analysis of these examples, however, reveals one more geometrical way to produce the locus configurations. 
Let $\mathfrak{S}$ be any affine configuration of hyperplanes in $\mathbf{C}^{n}$. Let's imbed $\mathbf{C}^{n}$ in $\mathbf{C}^{n+2}$ in the following way: $x=\left(x_{1}, \ldots, x_{n}\right) \rightarrow\left(x_{1}, \ldots, x_{n}, 1,0\right)$. For any hyperplane $\Pi$ in $\mathbf{C}^{n}$ let's define the hyperplane $\widetilde{\Pi}$ in $\mathbf{C}^{n+2}$ as a linear span of $\Pi \subset \mathbf{C}^{n} \subset \mathbf{C}^{n+2}$ and the isotropic vector $e=(0, \ldots, 0,1, i)$. If $(\alpha, x)+c=0$ is the equation of $\Pi$ in $\mathbf{C}^{n}$ then the corresponding equation of $\widetilde{\Pi}$ will be $(\alpha, x)+c\left(x_{n+1}+i x_{n+2}\right)=0$.

Corresponding configuration $\widetilde{\mathfrak{S}}$ in $\mathbf{C}^{n+2}$ we will call as isotropic projectivisation of $\mathfrak{S}$.

Theorem 5.4. The isotropic projectivisation of an affine locus configuration $\mathfrak{S}$ in $\mathbf{C}^{n}$ is a linear locus configuration $\widetilde{\mathfrak{S}}$ in $\mathbf{C}^{n+2}$.

Proof. We shall check the first of the locus equations for $\widetilde{\mathfrak{S}}$, the others can be checked in the same way. So, we need to prove that on a hyperplane $\left(\alpha_{s}, x\right)+c_{s}\left(x_{n+1}+i x_{n+2}\right)=0$ the following identity holds:

$$
\sum_{j \neq s} \frac{m_{j}\left(m_{j}+1\right)\left(\tilde{\alpha}_{j}, \tilde{\alpha}_{j}\right)\left(\tilde{\alpha}_{s}, \tilde{\alpha}_{j}\right)}{\left(\left(\alpha_{j}, x\right)+c_{j}\left(x_{n+1}+i x_{n+2}\right)\right)^{3}} \equiv 0
$$

where $\tilde{\alpha}_{j}$ denotes the normal vector of the hyperplane $\widetilde{\Pi}_{j} \subset \mathbf{C}^{n+2}$. If $\widetilde{\Pi}_{j} \subset \mathbf{C}^{n}$ has the the normal vector $\alpha_{j}=\left(\alpha_{j}^{1}, \ldots, \alpha_{j}^{n}\right)$, then $\tilde{\alpha}_{j}$ is the vector $\left(\alpha_{j}^{1}, \ldots, \alpha_{j}^{n}, c_{j}, i c_{j}\right)$. ¿From that we immediately see that $\left(\tilde{\alpha}_{j}, \tilde{\alpha}_{j}\right)=\left(\alpha_{j}, \alpha_{j}\right)$ and $\left(\tilde{\alpha}_{s}, \tilde{\alpha}_{j}\right)=\left(\alpha_{s}, \alpha_{j}\right)$. Now since $\lambda=x_{n+1}+i x_{n+2} \neq 0$ almost everywhere on the hyperplane $\left(\alpha_{s}, x\right)+c_{s}\left(x_{n+1}+i x_{n+2}\right)=0$ we come to the identity

$$
\sum_{j \neq s} \frac{m_{j}\left(m_{j}+1\right)\left(\alpha_{j}, \alpha_{j}\right)\left(\alpha_{s}, \alpha_{j}\right)}{\left(\left(\alpha_{j}, x\right)+c_{j} \lambda\right)^{3}} \equiv 0
$$

for $\left(\alpha_{s}, x\right)+c_{s} \lambda=0$. But this identity after the rescaling $x \rightarrow \lambda x$ takes the form

$$
\sum_{j \neq s} \frac{m_{j}\left(m_{j}+1\right)\left(\alpha_{j}, \alpha_{j}\right)\left(\alpha_{s}, \alpha_{j}\right)}{\left(\left(\alpha_{j}, x\right)+c_{j}\right)^{3}} \equiv 0 \quad \text { for } \quad\left(\alpha_{s}, x\right)+c_{s}=0
$$

which is exactly the first locus equation for the configuration $\mathfrak{S}$.

Example. Let $\mathfrak{S}$ be a direct sum of three-point one-dimensional configurations with the corresponding potential

$$
u\left(x_{1}, \ldots, x_{n}\right)=\sum_{i=1}^{n} \frac{6 x_{i}^{4}-12 \tau_{i} x_{i}}{\left(x_{i}^{3}+\tau_{i}\right)^{2}} .
$$

Then after the isotropic projectivisation we obtain the locus configuration with the potential of the form (cf. [16]):

$$
\tilde{u}\left(x_{1}, \ldots, x_{n+2}\right)=\sum_{j=1}^{n} \frac{6 x_{j}^{4}-12 \tau_{j}\left(x_{n+1}+i x_{n+2}\right)^{3} x_{j}}{\left(x_{j}^{3}+\tau_{j}\left(x_{n+1}+i x_{n+2}\right)^{3}\right)^{2}} .
$$

In order to obtain a more general Berest-Winternitz's potential [16]

$$
\tilde{u}\left(x_{1}, \ldots, x_{n+2}\right)=\sum_{j=1}^{n} \frac{6 x_{j}^{4}-12 \tau_{j}\left(x_{n+1}+i x_{n+2}+c_{j}\right)^{3} x_{j}}{\left(x_{j}^{3}+\tau_{j}\left(x_{n+1}+i x_{n+2}+c_{j}\right)^{3}\right)^{2}}
$$


we should shift the pairwise-orthogonal triples of hyperplanes

$$
x_{j}+\tau_{j}^{\frac{1}{3}}\left(x_{n+1}+i x_{n+2}\right)=0 \quad(j=1, \ldots, n)
$$

by $c_{j}$ in $x_{n+1}$.

Remark. The BA function in this example can be obtained easily using the following general remark. If $\psi_{i}=R_{i}(k, x) \exp (k, x) \quad(i=1,2)$ are given by the formula (40) for two orthogonal locus configurations $\mathfrak{S}_{1}$ and $\mathfrak{S}_{2}$ then the function $\psi=R_{1} R_{2} \exp (k, x)$ will correspond to the locus configuration $\mathfrak{S}=\mathfrak{S}_{1} \cup \mathfrak{S}_{2}$. This is clear from the structure of the formula (40).

Thus, iterating such geometric procedures one can construct many new affine locus configurations. However, all of them are degenerate in the following sense. Let $V(\mathfrak{S})$ be the linear space of the normals to all the hyperplanes in $\mathfrak{S}$. We call $\mathfrak{S}$ degenerate if the restriction of the complex Euclidean form on $V(\mathfrak{S})$ is degenerate.

For a degenerate affine configuration one can define the following isotropic reduction procedure, which is inverse to the isotropic projectivisation.

Let $K$ be the kernel of the restriction of the Euclidean form onto $V(\mathfrak{S})$. Consider the orthogonal complement $V^{\perp}$ of $V$ in $\mathbf{C}^{n}$ and choose a subspace $L$ such that

$$
V+V^{\perp}=K \oplus L
$$

By an isotropic reduction of the degenerate configuration $\mathfrak{S}$ we shall mean the configuration $\mathfrak{S} \cap\{a+L\}$, where $\{a+L\}$ is a shift of $L$ by a generic vector $a \in \mathbf{C}^{n}$.

Theorem 5.5. An isotropic reduction of a degenerate locus configuration is a nondegenerate locus configuration.

The proof is similar to the case of isotropic projectivisation.

These results may be interpreted in two ways. First, we can say that any affine locus configuration is a result of the isotropic reduction of some (degenerate) linear configuration. So, the classification problem for affine locus configurations reduces to the linear case. On the other hand, as we have shown, to classify all locus configurations it is sufficient to consider non-degenerate configurations only. Moreover, we can consider irreducible configurations only, i.e. exclude the unions of orthogonal subconfigurations. At the moment all the known non-degenerate irreducible locus configurations are linear or one-dimensional. It may well be the only possible examples.

The following general result clarifies the geometrical structure of affine locus configurations.

Theorem 5.6. Any affine locus configuration $\mathfrak{S}$ has the following properties:

(1) for each point $x_{0} \in \mathbf{C}^{n}$ the subset $\mathfrak{S}_{x_{0}} \subseteq \mathfrak{S}$ of the hyperplanes passing through $x_{0}$ form a linear locus configuration;

(2) for each hyperplane $\Pi \in \Sigma$ the subset $\mathfrak{S}(\Pi) \subseteq \mathcal{A}$ of the hyperplanes parallel to $\Pi$ forms an extended one-dimensional locus configuration.

Conversely, any affine configuration with properties (1), (2) belongs to the locus.

Proof. (1) Let's consider the locus equations for some hyperplane $\Pi_{i}:\left(\alpha_{i}, x\right)+c_{i}=0$ passing through $x_{0}$ :

$$
\sum_{j \neq i} \frac{m_{j}\left(m_{j}+1\right)\left(\alpha_{j}, \alpha_{j}\right)\left(\alpha_{i}, \alpha_{j}\right)^{2 s-1}}{\left(\left(\alpha_{j}, x\right)+c_{j}\right)^{2 s+1}} \equiv 0 \quad \text { for } \quad x \in \Pi_{i},
$$


$s=1, \ldots, m_{i}$.

Now take $x=x_{0}+y$, then $x \in \Pi_{i}$ iff $\left(\alpha_{i}, y\right)=0$ and we have the following relation:

$$
\sum_{\substack{j: x_{0} \in \Pi_{j} \\ j \neq i}} \frac{m_{j}\left(m_{j}+1\right)\left(\alpha_{j}, \alpha_{j}\right)\left(\alpha_{i}, \alpha_{j}\right)^{2 s-1}}{\left(\alpha_{j}, y\right)^{2 s+1}}+\sum_{k: x_{0} \notin \Pi_{k}} \frac{m_{k}\left(m_{k}+1\right)\left(\alpha_{k}, \alpha_{k}\right)\left(\alpha_{i}, \alpha_{k}\right)^{2 s-1}}{\left(\left(\alpha_{k}, x_{0}\right)+c_{k}+\left(\alpha_{k}, y\right)\right)^{2 s+1}} \equiv 0
$$

for all $y$ such that $\left(\alpha_{i}, y\right)=0$. Since the second sum is regular at $y=0$, the first sum should vanish on the hyperplane $\left(\alpha_{i}, y\right)=0$. Thus, we obtain linear locus equation for the configuration $\mathfrak{S}_{x_{0}}$.

(2) To prove the second property, let's divide all the hyperplanes which are non-parallel to $\Pi$ into the subgroups in the following way: $\Pi^{\prime}$ and $\Pi^{\prime \prime}$ belong to the same group if and only if their intersection is contained in $\Pi$. Then in each group the sum of the corresponding terms in (50) should vanish due to the property (1). The remaining terms is exactly the locus equation for the set of parallel planes $\mathfrak{S}(\Pi)$.

The converse statement now is clear.

We conclude this section by some negative results about locus configurations in $\mathbf{R}^{n}$.

Theorem 5.7. For any locus configuration in the real plane there exists a point all the lines pass through.

Proof. First we note that parallel lines cannot appear in locus configurations in $\mathbf{R}^{2}$. Indeed, the subset of parallel lines according to the previous theorem must give a real solution for the one-dimensional locus equations, which is impossible.

Now let's fix some terminology: by vertices we will mean the intersection points for the lines from the configuration and by a ray - any ray from the configuration with the origin at some vertex (some rays may contain other vertices). Let's choose an orientation on the plane. This allows us to determine the oriented angle $\varphi\left(l_{1}, l_{2}\right)$ between the ordered pair of rays $l_{1}, l_{2}$, which varies from $-\pi$ to $\pi$. We need the following property of the locus configurations in $\mathbf{R}^{2}$ :

Lemma 1. For each ray $l_{1}$ from the locus configuration in $\mathbf{R}^{2}$ there exists another ray $l_{2}$ with the same vertex and acute angle between $l_{1}$ and $l_{2}$ :

$$
0<\varphi\left(l_{1}, l_{2}\right) \leq \frac{\pi}{2}
$$

Similarly, there exists a ray $l_{3}$ with the same vertex such that $-\frac{\pi}{2} \leq \varphi\left(l_{1}, l_{3}\right)<0$.

Proof of the lemma follows from the linear locus equations (27) for the lines passing through a given vertex: it's clear that the sign of each term in it depends only on the sign of the cotangent of the oriented angle between $\alpha$ and $\beta$.

Lemma 2. Let $l_{1}$ and $l_{2}$ be chosen as in Lemma 1. Then if $l_{1}$ contains another vertex of the configuration, the same is true for $l_{2}$.

The proof follows from simple geometrical considerations.

Let's consider now any vertex and all the rays of our configuration outgoing from this vertex. As it easily follows from the lemmas we have only two possibilities:

1) there are no other vertices on these rays or

2) there is at least one more vertex on each ray.

Since we have a finite number of vertices, we obtain immediately that our configuration has only one vertex. Theorem is proven. 
The same is probably true in $\mathbf{R}^{n}$ but at the moment we can prove this only in the special case when all the multiplicities are equal.

Theorem 5.8. Any affine locus configuration in $\mathbf{R}^{n}$ with equal multiplicities is a linear Coxeter configuration.

Proof. It's sufficient to prove that the configuration must be symmetric with respect to each its hyperplane. Since the parallel hyperplanes cannot appear in a real locus configuration, the statement follows from the theorem 5.6 and the corollary 4.7.

\section{Locus configurations and Huygens' Principle.}

Let us consider a linear hyperbolic equation

$$
\mathcal{L} \varphi(x)=0, \quad \mathcal{L}=\square_{N+1}+u(x),
$$

where $\square_{N+1}$ is the D'Alembert operator, $\square_{N+1}=\frac{\partial^{2}}{\partial x_{0}^{2}}-\frac{\partial^{2}}{\partial x_{1}^{2}}-\ldots-\frac{\partial^{2}}{\partial x_{N}^{2}}$.

We say after J.Hadamard [30] that it satisfies Huygens' Principle (HP) if its fundamental solution is located on the characteristic conoid, i.e. this solution vanishes in the conoid's complement.

Hadamard found some criterion for HP to be satisfied in terms of the so-called Hadamard's coefficients $U_{\nu}(x, \xi)$. They are uniquely determined by the following system of equations

$$
\sum_{i=0}^{N}\left(x_{i}-\xi_{i}\right) \frac{\partial U_{\nu}}{\partial x_{i}}+\nu U_{\nu}=-\frac{1}{2} \mathcal{L}\left(U_{\nu-1}\right)
$$

and the conditions that $U_{0}(x, \xi) \equiv 1$ and $U_{\nu}(x, \xi)$ are regular at $x=\xi$. These coefficients are symmetric with respect to $x$ and $\xi: U_{\nu}(x, \xi)=U_{\nu}(\xi, x)$ (for the details see the book [31]).

Hadamard proved that the equation (51) satisfies Huygens' Principle if and only if $N$ is odd and $\left.U_{\nu}\right|_{\Gamma}=0$ for $\nu \geq \frac{N-1}{2}$, where $\Gamma=\left\{(x, \xi):\left(x_{0}-\xi_{0}\right)^{2}-\sum_{i=1}^{N}\left(x_{i}-\xi_{i}\right)^{2}=0\right\}$ is the characteristic conoid. For the case when potential $u$ (and, as a corollary, all the Hadamard's coefficients $U_{\nu}$ ) does not depend on at least one of the coordinates (say, $x_{0}$ ), the Hadamard's criterion is equivalent to the condition $U_{\frac{N-1}{2}} \equiv 0$.

We consider the Hadamard's problem of description of all huygensian equations of the form:

$$
\left(\square_{N+1}+u\left(x_{1}, \ldots, x_{N}\right)\right) \varphi=0
$$

In fact, in our case for any locus configuration in $\mathbf{C}^{n}$ the corresponding potential will depend only on the first $n$ coordinates : $u=u\left(x_{1}, \ldots, x_{n}\right), n \leq N$.

It turns out that huygensian equations of the form (53) are closely related to the locus configurations. For the linear locus configuration in $\mathbf{C}^{n}$ the corresponding potential

$$
u(x)=\sum_{\alpha \in \mathcal{A}} \frac{m_{\alpha}\left(m_{\alpha}+1\right)(\alpha, \alpha)}{(\alpha, x)^{2}} .
$$

is homogeneous of degree -2 . 
Theorem 6.1. For any real potential $u\left(x_{1}, \ldots, x_{n}\right)$ related to a linear locus configuration the hyperbolic equation (53) satisfies $H P$ if $N$ is odd and $N \geq 2 \sum_{\alpha \in \mathcal{A}} m_{\alpha}+3$. In that case the fundamental solution can be expressed via BA function.

Conversely, if the hyperbolic equation (53) with homogeneous potential $u(x): u(\lambda x)=$ $\lambda^{-2} u(x)$ satisfies HP and all the Hadamard's coefficients are rational functions, then the potential $u(x)$ must have the form (54) for some linear locus configuration.

Proof. The proof of the first statement repeats the arguments of the paper [10], where this result has been proven in the Coxeter case. It is based on the following relation between BA function and Hadamard's coefficients. If we have the Baker-Akhiezer function $\psi$ of the form (7), we can present it in the form

$$
\psi(\xi, x)=\left(U_{0}(\xi, x)+U_{1}(\xi, x)+\ldots+U_{M}(\xi, x)\right) e^{(\xi, x)},
$$

where $U_{0}=1, U_{\nu}(x, \xi)$ is homogeneous of degree $-\nu$ in $\xi, M=\operatorname{deg} A(k)=\sum_{\alpha \in \mathcal{A}} m_{\alpha}$. Since $\psi$ is symmetric in $\xi$ and $x$ (theorem 2.3), $U_{\nu}$ has the same degree in $x$. From the Schrödinger equation (9) for $\psi, L \psi=-\xi^{2} \psi, L=-\Delta+u(x)$, we obtain:

$$
-2 \sum_{i=1}^{n} \xi_{i} \frac{\partial}{\partial x_{i}} U_{\nu}+L\left[U_{\nu-1}\right]=0 \quad\left(\nu=1, \ldots, M+1 \text { with } U_{M+1}=0\right) .
$$

Since $U_{\nu}$ are homogeneous in $x$ this implies the relations (52), so $U_{\nu}$ coincide with the Hadamard's coefficients. Now since $U_{M+1}=0$ the Hadamard's criterion guarantees HP if $N \geq 2 M+3$. Notice that it gives also the explicit formula for the Hadamard's coefficients and the fundamental solution for (51) (see for the details [10]).

Conversely, from the chain (52) for the Hadamard's coefficients $U_{\nu}(x, \xi)$ for the homogeneous potential $u$ it follows that $U_{\nu}$ are also homogeneous in $x$ (and, therefore, in $\xi)$ :

$$
U_{\nu}(\lambda x, \xi)=\lambda^{-\nu} U_{\nu}(x, \xi)=U_{\nu}(x, \lambda \xi) .
$$

This can be proven by the same calculation as in lemma 1 from [12], where the case $n=2$ was considered. Let's now consider the function $\psi$ defined by the formula (55). Then, from the Hadamard chain (52) and homogeneity of $U_{\nu}$ it follows in the same way as above that $\psi$ satisfies the Schrödinger equation

$$
\left(-\Delta_{N}+u(x)\right) \psi=-\xi^{2} \psi
$$

Notice that the potential $u(x)$ must be rational since all the Hadamard's coefficients are supposed to be rational. This follows from the first equation of the Hadamard's chain (52). Now using the theorems 2.1 and 2.2 and the fact that $u(x)$ is homogeneous of degree $(-2)$ we conclude that $u(x)$ has the form (54) for some locus configuration.

Remark. In the case when $n=2$ i.e. $u=u\left(x_{1}, x_{2}\right)$, a stronger result (namely, without the assumption that the Hadamard's coefficients are rational) follows from the results by Yu.Berest and I.Lutsenko [11], [12].

Now let's consider an arbitrary (affine) locus configuration $\mathfrak{S}$ such that the corresponding potential $u(x)$ given by the formula (38) is real for real $x$. This is equivalent to the condition $\mathfrak{S}=\bar{\Sigma}$ where $\overline{\mathfrak{S}}$ is a natural complex conjugation of a configuration $\mathfrak{S}$. The following result generalises the Theorem 6.1 for the general (affine) locus configurations. 
Theorem 6.2. For any affine locus configuration $\mathfrak{S} \subset \mathbf{C}^{n}$ with $\mathfrak{S}=\bar{\Sigma}$ the corresponding hyperbolic equation (53) satisfies Huygens' Principle if $N$ is odd and large enough: $N \geq 2 M+3, M=\sum_{j=1}^{K} m_{j}$.

Conversely, if the equation (53) satisfies Huygens' Principle and all the Hadamard's coefficients are rational functions, then the potential $u(x)$ must be of the form (38) for some affine locus configuration.

Proof. The first part of this theorem can be derived from the theorem 5.1 and the results by Yu.Berest [34] (see also [19]). We would like, however, to present here another, more illuminating proof. It is based on a different idea which will help us to prove the second part also. The idea is to reduce the affine case to the linear one using the isotropic projectivisation procedure.

The main observation is encapsulated in the following lemma. Let $U_{\nu}(x, \xi) \quad(\nu=$ $0,1, \ldots)$ be some analytic functions of $2 n$ variables $x=\left(x_{1}, \ldots, x_{n}\right), \xi=\left(\xi_{1}, \ldots, \xi_{n}\right)$ which satisfy the equations (52) with some potential $u(x)$. Let's define now the new functions depending on $\tilde{x}=\left(x_{1}, \ldots, x_{n}, x_{n+1}, x_{n+2}\right)$ and $\tilde{\xi}=\left(\xi_{1}, \ldots, \xi_{n}, \xi_{n+1}, \xi_{n+2}\right)$ :

$$
\widetilde{U}_{\nu}(\tilde{x}, \tilde{\xi})=\left(x_{n+1}+i x_{n+2}\right)^{-\nu}\left(\xi_{n+1}+i \xi_{n+2}\right)^{-\nu} U_{\nu}\left(\frac{x}{x_{n+1}+i x_{n+2}}, \frac{\xi}{\xi_{n+1}+i \xi_{n+2}}\right)
$$

and

$$
\tilde{u}(\tilde{x})=\left(x_{n+1}+i x_{n+2}\right)^{-2} u\left(\frac{x}{x_{n+1}+i x_{n+2}}\right) .
$$

Lemma. The relations (52) for $U_{\nu}(x, \xi)$ and $u(x)$ are equivalent to the similar relations in $\tilde{x}, \tilde{\xi}$ for $\widetilde{U}_{\nu}(\tilde{x}, \tilde{\xi})$ and $\tilde{u}(\tilde{x})$ defined by the formulas (56) and (57).

The proof is straightforward.

Now suppose that we have the real potential $u(x)$ related to some affine locus configuration $\mathfrak{S}=\overline{\mathfrak{S}} \subset \mathbf{C}^{n}$. Then the potential $\tilde{u}(\tilde{x})$ defined by (57) corresponds to some locus configuration $\widetilde{\mathfrak{S}} \subset \mathbf{C}^{n+2}$ which is exactly the result of the isotropic projectivisation defined in the previous section (see Theorem 5.4). Thus, according to the Theorem 3.1 the corresponding Schrödinger operator $\widetilde{L}=-\Delta_{n+2}+\tilde{u}(\underset{x}{\tilde{x}})$ in $\mathbf{C}^{n+2}$ has the BA function $\widetilde{\psi}(\tilde{\xi}, \tilde{x})$ which is given by the formula $(28)$. Therefore, $\widetilde{\psi}$ can be presented in the form analogous to $(55)$,

$$
\widetilde{\psi}(\tilde{\xi}, \tilde{x})=\left(\widetilde{U}_{0}(\tilde{\xi}, \tilde{x})+\widetilde{U}_{1}(\tilde{\xi}, \tilde{x})+\ldots+\widetilde{U}_{M}(\tilde{\xi}, \tilde{x})\right) e^{(\tilde{\xi}, \tilde{x})},
$$

where $\widetilde{U}_{0}=1$ and the components $\widetilde{U}_{\nu}(\tilde{x}, \tilde{\xi})$ are homogeneous of degree $-\nu$ in $\tilde{\xi}$ and $\tilde{x}$, non-singular for $\tilde{x}=\tilde{\xi}$ and satisfy the relations $(52)$ in $\tilde{x}, \tilde{\xi}$ with the potential $\tilde{u}(\tilde{x})$. Now let's consider their restriction for $x_{n+1}+i x_{n+2}=\xi_{n+1}+i \xi_{n+2}=1$,

$$
U_{\nu}(x, \xi)=\left.\widetilde{U}_{\nu}(\tilde{x}, \tilde{\xi})\right|_{\substack{x_{n+1}+i x_{n+2}=1 \\ \xi_{n+1}+i \xi_{n+2}=1}}
$$

We claim that the formula (59) determines the Hadamard's coefficients for the initial potential $u(x)$.

First of all, let's notice that this formula really determines some functions of $x, \xi$ only. This can be derived directly from the formula (28). Indeed, it's easy to see from the inductive procedure (29) that the pre-exponent in the BA function (28) is a linear 
combination of the "monomial" terms $\prod_{\widetilde{\mathcal{A}}}(\alpha, x)^{p_{\alpha}}(\alpha, k)^{q_{\alpha}}$ with some integers $p_{\alpha}, q_{\alpha}$. Thus, $x_{n+1}, x_{n+2}, \xi_{n+1}, \xi_{n+2}$ will enter in $\widetilde{\psi}$ only as combinations $x_{n+1}+i x_{n+2}$ and $\xi_{n+1}+$ $i \xi_{n+2}$. This means that the coefficients $U_{\nu}$ defined by (59) indeed do not depend on $x_{n+1}, x_{n+2}, \xi_{n+1}, \xi_{n+2}$. As a corollary of the homogeneity of $\widetilde{U}_{\nu}$ in $\tilde{x}$ and $\tilde{\xi}$ we may invert the formula (59) and obtain that $\widetilde{U}_{\nu}$ are related to $U_{\nu}$ by the formula (56). Now using the lemma we get the equations (52) for $U_{\nu}$. It is clear then from (59) that $U_{0}=1$ and $U_{\nu}$ are non-singular when $x=\xi$. The last remark is that the procedure (59) gives us the real-valued functions $U_{\nu}$ of $x, \xi \in \mathbf{R}^{n}$ in the case when the initial potential $u(x)$ is real, $\mathfrak{S}=\overline{\mathfrak{S}}$.

So, for any affine locus configuration we constructed the Hadamard's coefficients $U_{\nu}$ for the corresponding hyperbolic equation (53), and $U_{M+1}=0$. Applying the Hadamard's criterion, we obtain the first part of the theorem.

To prove the inverse statement, we suppose that the hyperbolic equation (53) is huygensian and has rational Hadamard's coefficients $U_{\nu}$ with $U_{M+1}=0$. In that case we can define the homogeneous functions $\widetilde{U}_{\nu}(\tilde{x}, \tilde{\xi})$ by the formula (56). According to the lemma, they obey the equations (52) with the homogeneous potential (57). Then in the same way as in the Theorem 6.1, we conclude that the function (58) satisfies the Schrödinger equation $\widetilde{L} \widetilde{\psi}=-\xi^{2} \psi$ with $\widetilde{L}=-\Delta_{n+2}+\tilde{u}(\tilde{x})$. Now using the Theorem 2.2 in the same way as in the theorem 6.1 we deduce that the potential $\tilde{u}(\tilde{x})$ must correspond to some (linear) locus configuration $\widetilde{\mathfrak{S}}$ of non-isotropic hyperplanes in $\mathbf{C}^{n+2}$. But in that case the initial potential $u(x)$ (see the formula (57)) will correspond to the isotropic reduction $\mathfrak{S}$ of $\widetilde{\mathfrak{S}}$ which should satisfy the locus equations due to the theorem 5.5. The theorem is proven.

Remark. We have assumed that the potential $u$ of the hyperbolic equation does not depend on $x_{0}$, but essentially we have used only the fact that the sequence of the Hadamard's coefficients terminates at some step $M$. Actually all the results of this section can be generalised formally for any equation of the form (51) (even with the complex potential), which possesses the last property. In that case the singularities of the potential should satisfy the locus equations in $\mathbf{C}^{N, 1}$ with the complex Euclidean structure defined by the metrics $\operatorname{diag}(-1,1, \ldots, 1)$.

We conjecture that any hyperbolic equation $\left(\square_{N+1}+u(x)\right) \varphi=0$ with terminating sequence of the Hadamard's coefficients has a rational potential $u(x)$ which corresponds to some locus configuration in $\mathbf{C}^{N, 1}$. We have proved this under the assumption that the Hadamard's coefficients are rational. The proof of this conjecture would lead to the solution of the famous Hadamard's problem in the class (53). Until now this problem is solved only when $u$ depends on one of the coordinates (K.Stellmacher, J.Lagnese [17]) and when $u$ is homogeneous and depends on two of the coordinates (Yu.Berest [12]).

\section{Some other relations and generalisations.}

7.1. The Baker-Akhiezer function $\psi(k, x)$ related to an equipped configuration has the following remarkable property: it satisfies a system of differential equations not only in $x$ but also in $k$-variables. Corresponding bispectral property of the one-dimensional BA function has been observed in the fundamental paper by Duistermaat and Grünbaum [14]. 
Let $\psi(k, x)$ be a BA function related to some equipped configuration $\mathfrak{A}, \mathfrak{S}$ be the corresponding dual configuration of the poles of the potential $u(x)$ given by (38).

Let $\mathcal{R}$ be the ring of polynomials defined in the Theorem 5.3. Define also the dual ring $\mathcal{S}$ as the ring of all polynomials $q(x)$ in $x$, satisfying the relations

$$
\left.\left(\alpha_{s}, \frac{\partial}{\partial x}\right)^{2 j-1}[q(x)]\right|_{\left(\alpha_{s}, x\right)+c_{s}=0} \equiv 0
$$

for all $j=1,2, \ldots, m_{s}$ and for all the hyperplanes of the configuration $\mathfrak{S}$.

Theorem 7.1. For any $p(k) \in \mathcal{R}$ and $q(x) \in \mathcal{S}$ there exist the differential operators $L_{p}(x, \partial / \partial x)$ and $M_{q}(k, \partial / \partial k)$ such that the BA function $\psi(k, x)$ satisfies the following bispectral problem:

$$
\left\{\begin{array}{l}
L_{p}(x, \partial / \partial x) \psi(k, x)=p(k) \psi(k, x) \\
M_{q}(k, \partial / \partial k) \psi(k, x)=q(x) \psi(k, x)
\end{array}\right.
$$

The existence of the operator $L_{p}(x, \partial / \partial x)$ is claimed in the Theorem 5.3. The existence of $M_{q}(k, \partial / \partial k)$ follows from the characterisation of $\psi$ by its analytic properties in $x$. Namely, one can show that the BA function $\psi(k, x)$ is the unique function of the form

$$
\psi=\frac{B(x)+\ldots}{B(x)} e^{(k, x)},
$$

where $B(x)=\prod_{s=1}^{N}\left(\left(\alpha_{s}, x\right)+c_{s}\right)^{m_{s}}$ and the dots denote the polynomial in $x$ of a smaller degree, such that the following conditions are fulfiled:

$$
\left.\left(\alpha_{s}, \frac{\partial}{\partial x}\right)^{2 j-1}\left[\left(\left(\alpha_{s}, x\right)+c_{s}\right)^{m_{s}} \psi\right]\right|_{\left(\alpha_{s}, x\right)+c_{s}=0} \equiv 0
$$

for each $j=1,2, \ldots, m_{s}$ and $s=1, \ldots, N$. The fact that the BA function satisfies these conditions follows from the Schrödinger equation (45) and the theorem 2.2.

7.2. Similar approach can be developed for the trigonometric versions of our Schrödinger operators (1). As well as in the rational case discussed in the present paper, the axiomatics of [4] has to be amended in order to cover the most general case. We intend to discuss such axiomatics in a separate paper. The corresponding locus conditions have been described in [21]. The bispectral property for the corresponding BA functions results in difference operators in the spectral parameter, which can be viewed as deformations of the rational Ruijsenaars and Macdonald operators (see [35]).

7.3. The most of the results of this paper can be generalised to the case when the potential $u(x)$ of the Schrödinger operator is a matrix-valued function. The locus equations for that case in dimension 1 have been described in [36]. Multidimensional case is considered in [37].

Acknowledgements. This work was partially supported by Russian Fundamental Research Fund (grants 96-01-01404, 96-15-96027, 96-15-96037) and INTAS (grant 96-0770). O.Ch. was supported also by the Royal Society postdoctoral fellowship during 1998, which is highly acknowledged. O.Ch. and M.F. are grateful to Loughborough University, UK, for the hospitality during the period this work was being completed. Finally, we would like to thank Yuri Berest for extremely fruitful discussions. 


\section{References}

[1] I.M.Krichever Methods of algebraic geometry in the theory of nonlinear equations. //Uspekhi Mat. Nauk, 1977, v.32(6), p.198-245.

[2] B.A.Dubrovin, V.B.Matveev, S.P.Novikov Nonlinear equations of Korteweg-de Vries type, finite-gap linear operators and abelian varieties. //Uspekhi Mat. Nauk, 1976, v.31(1), p.51-125.

[3] E.T.Whittaker, G.N.Watson A course of modern analysis. Cambridge University Press, 1963.

[4] O.A.Chalykh, A.P.Veselov Commutative rings of partial differential operators and Lie alrebras. //Commun. Math. Phys. 1990, v.126, p.597-611.

[5] A.P.Veselov, K.L.Styrkas, O.A.Chalykh Algebraic integrability for Schrodinger equation and finite reflection groups. //Theor. Math. Phys., 1993, v.94(2), p.253-275.

[6] M.A.Olshanetsky, A.M.Perelomov Quantum integrable systems related to Lie algebras. //Phys. Rep., 1983, v.94, p.313-404.

[7] G.J.Heckman A remark on the Dunkl differential-difference operators. //Prog. in Math., 1991, v.101, p.181-191.

[8] A.P.Veselov, M.V.Feigin, O.A.Chalykh New integrable deformations of quantum Calogero - Moser problem. //Russian Math. Surveys, 1996, v.51(3), p.185-186.

[9] O.A.Chalykh, M.V.Feigin, A.P.Veselov New integrable generalizations of CalogeroMoser quantum problem. //J. Math. Phys., 1998, v.39(2), p.695-703.

[10] Yu.Yu.Berest, A.P.Veselov Hadamard's problem and Coxeter groups: new examples of the huygensian equations. //Funct. Anal. Appl., 1994, v.28(1), p.3-15.

[11] Yu. Yu. Berest, I. M. Lutsenko Huygens' principle in Minkowski spaces and soliton solutions of the Korteweg-de Vries equation. //Commun. Math. Phys., 1997, v.190, p.113-132.

[12] Yu.Yu.Berest Solution of a Restricted Hadamard's Problem in Minkowski Spaces. //Comm.Pure Appl.Math., 1997, v.50(10), p.1019-1052.

[13] H.Airault, H.P.McKean, J.Moser Rational and elliptic solutions of the Korteweg-de Vries equation and a related many-body problem. //Comm. Pure Appl. Math., 1977, v.30, p.95-178.

[14] J.J.Duistermaat, F.A.Grünbaum Differential equations with the spectral parameter. //Comm.Math.Phys., 1986, v.103, p.177-240.

[15] M.Adler, J.Moser On a class of polynomials connected with the Korteweg-de Vries equation. //Commun. Math. Phys., 1978, v.61, p.1-30. 
[16] Yu.Berest, P.Winternitz Huygens' principle and separation of variables., Preprint CRM-2379 (1996) (to appear in Comm.Math.Phys.).

[17] J.E.Lagnese, K.L.Stellmacher A method of generating classes of Huygens' operators. //J. Math. \& Mech., 1967, v.17(5), p.461-472.

[18] Yu.Berest Huygens' principle and the bispectral problem. // CRM Proceedings and Lecture Notes, 1998, v.14, p.11-30.

[19] Yu.Yu.Berest, A.P.Veselov On the singularities of the potentials of exactly solvable Schrödinger operators and Hadamard's problem. //Russian Math. Surveys, 1998, v.53(1), p.211-212.

[20] Yu.Berest, A.Veselov On the Structure of Singularities of Integrable Schrödinger Operators. Submitted to Letters in Math. Physics.

[21] O.A.Chalykh Darboux transformations for multidimensional Schrödinger operators. //Russian Math. Surveys, 1998, v.53(2), p.167-168.

[22] F.A.Berezin Laplace operators on semisimple Lie groups. //Proc. Moscow Math. Soc., 1957, v.6, p.371-463.

[23] N. Bourbaki Groupes et algèbres de Lie. Chap. VI, Masson, 1981

[24] F.Calogero Solution of the one-dimensional $n$-body problem with quadratic and/or inversely quadratic pair potential. //J.Math.Phys., 1971, v.12, p.419-436.

[25] C.F.Dunkl Differential-difference operators associated to reflection groups. //Trans. AMS., 1989, v.311, p.167-183.

[26] A.M.Cohen Finite complex reflection groups //Ann.scient.Ec.Norm.Sup., 1976, ser.4, v.9, p.379-436.

[27] E.L.Ince Ordinary differential equations. Dover publications, 1956.

[28] T.Oshima A definition of boundary values of solutions of partial differential equations with regular singularities. //Publications of the RIMS, Kyoto Univ., 1983, v.19, p.1203-1230.

[29] M.M.Crum Associated Sturm-Liouville systems. //Quart. J.Math., 1955, ser.2(6), p.121-126.

[30] J.Hadamard Lectures on Cauchy's Problem in Linear Partial Differential Equations. New Haven: Yale Univ. Press, 1923.

[31] P.Günther Huygens' Principle and Hyperbolic Equations. Boston: Acad.Press,1988.

[32] J.L.Burchnall, T.W.Chaundy A set of differential equations which can be solved by polynomials. //Proc. London Math. Soc., 1929-30, v.30, p.401-414.

[33] G.Segal, G.Wilson Loop groups and equations of KdV type.//Publ. IHES, 1985, v.61, p.5-65. 
[34] Yu.Berest Hierarchies of Huygens' operators and Hadamard's conjecture. //Acta Appl.Math., 1998, v.53, p.125-185.

[35] O.A.Chalykh Duality of the generalized Calogero and Ruijsenaars problems. //Russian Math. Surveys, 1997, v.52(6), p.191-192.

[36] V.M.Goncharenko, A.P.Veselov Monodromy of the matrix Schrödinger equations and Darboux transformations. //J.Phys. A: Math.Gen., 1998, v.31, p.5315-5326.

[37] O.A.Chalykh, V.M.Goncharenko, A.P.Veselov Multidimensional integrable Schrödinger operators with matrix potential. In preparation. 